\title{
Bifurcation analysis and chaos control in discrete-time modified Leslie-Gower prey harvesting model
}

\author{
Muhammad Bilal Ajaz ${ }^{1 *}$, Umer Saeed $^{2}$, Qamar Din $^{3}$, Irfan Ali $^{1}$ and Muhammad Israr Siddiqui ${ }^{2}$
}

"Correspondence:

m6a.bilal@gmail.com

${ }^{1}$ School of Natural Sciences,

National University of Sciences and

Technology, Islamabad, Pakistan

Full list of author information is

available at the end of the article

\section{Springer}

\begin{abstract}
We investigate the dynamical behavior of a modified Leslie-Gower prey-predator model with harvesting in prey population. In order to explore rich dynamics of the model, Euler approximation is implemented to obtain a discrete-time modified Leslie-Gower model. Existence of equilibria and their local asymptotic stabilities are carried out. Furthermore, with the help of bifurcation theory and center manifold theorem, existence and directions of period-doubling and Neimark-Sacker bifurcations are investigated at positive steady-state. In order to control chaos and bifurcations, the Ott-Grebogi-Yorke (OGY) method and the hybrid control strategy are introduced. Numerical simulations are also provided to illustrate the theoretical discussions.
\end{abstract}

Keywords: Modified Leslie-Gower model; Stability analysis; Period-doubling bifurcation; Neimark-Sacker bifurcation; Chaos control

\section{Introduction}

Discrete-time models administered by distinction conditions are much more suitable than the continuous ones when the population is of non-overlapping generations [1-4]. Due to large applications in nearly all fields of applied sciences, discrete dynamical systems are an effective point of research. Discrete dynamical systems show rich and complex dynamical properties, for example, transcritical bifurcation, flip bifurcation, Hopf bifurcation, and chaos [5-8]. To study the nonlinear systems and their ramifications, qualitative analysis of discrete dynamical systems is the main mathematical apparatus to use. Integrity of the discrete-time models adds significance in the research community. For an examination of the qualitative behavior of difference equations, an easy computational and graphical explanation tools of illustration are available, but the analytical analysis always keeps a tremendous task especially in the case of the complicated and amazingly varying difference equations [9-11]. The predator-prey co-operations are the foremost complex zones of the population environment. Their universal reality and extraordinary behavior have created the interest of scholars, ecologists, and mathematicians during the final few years [12]. An innovative result, i.e., Lotka-Volterra predator-prey model given by Lotka [13] and Volterra [14] independently, is the first and challenging mathematical model. The

(c) The Author(s) 2020. This article is licensed under a Creative Commons Attribution 4.0 International License, which permits use, sharing, adaptation, distribution and reproduction in any medium or format, as long as you give appropriate credit to the original author(s) and the source, provide a link to the Creative Commons licence, and indicate if changes were made. The images or other third party material in this article are included in the article's Creative Commons licence, unless indicated otherwise in a credit line to the material. If material is not included in the article's Creative Commons licence and your intended use is not permitted by statutory regulation or exceeds the permitted use, you will need to obtain permission directly from the copyright holder. To view a copy of this licence, visit http://creativecommons.org/licenses/by/4.0/. 
Lotka-Volterra predator-prey model has neglected many actual conditions and complications and was modeled in such a way that carries the reality. Leslie and Gower [15] suggested a predator-prey model, called the Leslie-Gower predator-prey model, in which the predator growth activity is clear-cut from the predator predation function. They presumed that the predator growth is interpreted by a function of the rate of predators and their prey. Many authors have employed this model to study the real global complications; for example, Wollkind, Logan, and Wollkind $[16,17]$ applied this system to model the predator-prey speck outbreak synergies on fruit trees in Washington state. Aziz and Okiye [18] set up and examined the modified LG model. Xiao and Huang [19] examined a predator-prey system with Holling type-IV functional response. Pattern formation and bifurcation analysis were discussed for a class of predator-prey system in [20]. Influence of fear factor on predator-prey dynamics with prey refuge was analyzed in [21, 22].

In biological mathematics, discrete-time models are used to examine the taxonomic group of organisms and species with the passage of time. Discrete-time population models are appropriate where the populations are non-overlapping and essentially remain constant over a generation. These models are best to describe the chaotic behavior of nonlinear dynamics $[23,24]$. Controlling chaos is an interesting topic in recent studies of nonlinear dynamical systems. Sometime bifurcation and chaotic behavior are highly unfavorable phenomena in dynamical systems because there may be an extinction of population due to chaos. So, controlling chaos by introducing new measures to the population is very important in the population dynamical systems. We are able to get chaos control by using different strategies, e.g., feedback control strategy, hybrid control technique, and poleplacement method. By using these techniques, one can delay, advance, or even eliminate the chaotic behavior due to emergence of bifurcation in dynamical systems.

Next, arguing as in [25], we consider the following class of predator-prey interaction with nonlinear harvesting in prey population and Holling type-IV functional response:

$$
\begin{aligned}
& \frac{d x}{d t}=x(1-x)-\frac{x y}{\frac{x^{2}}{\alpha}+x+\beta}-\frac{a x}{x+b}, \\
& \frac{d y}{d t}=c y\left(1-d \frac{y}{x}\right),
\end{aligned}
$$

where $x$ and $y$ are prey and predator density, respectively. All the parameters $a, b, c, d, \alpha$, and $\beta$ are positive constants. Moreover, $\alpha$ represents tolerance of the prey, $\beta$ denotes half saturation constant, $a$ is the catchability coefficient, $b$ represents the effort for harvesting, $c$ is intrinsic growth rate of the predator, and $d$ is the amount of prey required to support one predator at equilibrium.

The qualitative behavior of continuous system (1) has been investigated recently in [25]. In order to explore rich dynamical behavior of such predator-prey interaction, one may consider some discrete counterpart of system (1). For this, we apply Euler's approximation to system (1) as follows:

$$
\begin{aligned}
& x_{n+1}=x_{n}+h\left[x_{n}\left(1-x_{n}\right)-\frac{x_{n} y_{n}}{\frac{x_{n}^{2}}{\alpha}+x_{n}+\beta}-\frac{a x_{n}}{x_{n}+b}\right], \\
& y_{n+1}=y_{n}+h\left[c y_{n}\left(1-d \frac{y_{n}}{x_{n}}\right)\right] .
\end{aligned}
$$




\section{Stability analysis of equilibria}

The equilibria for system (2) are the solutions of the following system:

$$
\begin{aligned}
& x(1-x)-\frac{x y}{\frac{x^{2}}{\alpha}+x+\beta}-\frac{a x}{x+b}=0, \\
& y\left(1-d \frac{y}{x}\right)=0 .
\end{aligned}
$$

Then it follows that $\left(x_{1}, 0\right)$ and $\left(x_{2}, 0\right)$ are boundary equilibria of system (2), where $x_{1}$ and $x_{2}$ are real roots of the following quadratic equation:

$$
x^{2}+(b-1) x+a-b=0
$$

such that $x_{1}=\frac{1}{2}\left(1-b-\sqrt{(1+b)^{2}-4 a}\right), x_{2}=\frac{1}{2}\left(1-b+\sqrt{(1+b)^{2}-4 a}\right)$ and $(1+b)^{2}-4 a>0$. Moreover, assume that $0<b<1$ and $b<a<\frac{1}{4}(b+1)^{2}$, then both $x_{1}$ and $x_{2}$ are positive with $x_{1}<x_{2}$. Next, we take $x_{1,2}=\bar{x}$, then the Jacobian matrix of system (2) at boundary equilibrium points $(\bar{x}, 0)$ is computed as follows:

$$
J(\bar{x}, 0)=\left(\begin{array}{cc}
1+h-2 \bar{x} h-\frac{a b h}{(b+\bar{x})^{2}} & -\frac{h \alpha \bar{x}}{\bar{x}(\bar{x}+\alpha)+\alpha \beta} \\
0 & 1+c h
\end{array}\right) .
$$

Then $\lambda_{1}=1+h-2 \bar{x} h-\frac{a b h}{(b+\bar{x})^{2}}$ and $\lambda_{2}=1+c h$ are eigenvalues of $J(\bar{x}, 0)$ with $\lambda_{2}>1$. Therefore, both boundary equilibria $\left(x_{1}, 0\right)$ and $\left(x_{2}, 0\right)$ are unstable. Next, we are looking for interior equilibria of system (2). For this, the components of interior equilibrium $\left(x^{*}, y^{*}\right)$ are given as follows:

$$
y^{*}=\frac{1}{d} x^{*},
$$

where $x^{*}$ is a positive real root for the following quartic equation:

$$
A x^{4}+B x^{3}+C x^{2}+D x+E=0
$$

where $A=d, B=d(\alpha+b-1), C=d(\alpha(\beta-1)+a+b(\alpha-1))+\alpha, D=\alpha(a d+(b-1) \beta d-b d+b)$, and $E=\alpha \beta d(a-b)$. In order to see the existence of positive equilibria graphically, we consider nullclines defined by $y=\frac{x}{d}$ and $y=\left(1-x-\frac{a}{b+x}\right)\left(\beta+\frac{x^{2}}{\alpha}+x\right)$. Then Fig. 1 shows the existence of two distinct positive equilibria $(0.219259,0.609053)$ and $(0.714336,1.98427)$, and Fig. 2 reveals the existence of the unique positive equilibrium point $(0.734318,1.93242)$ for system (2).

Furthermore, the Jacobian matrix at positive equilibrium $\left(x^{*}, y^{*}\right)$ is given by

$$
J\left(x^{*}, y^{*}\right)=\left(\begin{array}{cc}
w_{11} & w_{12} \\
\frac{c h}{d} & 1-c h
\end{array}\right)
$$

where

$$
w_{11}:=1+h\left(\frac{a x^{*}}{\left(b+x^{*}\right)^{2}}+\frac{x^{*} y^{*}\left(1+\frac{2 x^{*}}{\alpha}\right)}{\left(\beta+x^{*}\left(\frac{x^{*}}{\alpha}+1\right)\right)^{2}}-x^{*}\right),
$$


Figure 1 Existence of two positive equilibria for $\alpha=0.054, \beta=0.06, a=0.07, b=0.05, d=0.36$

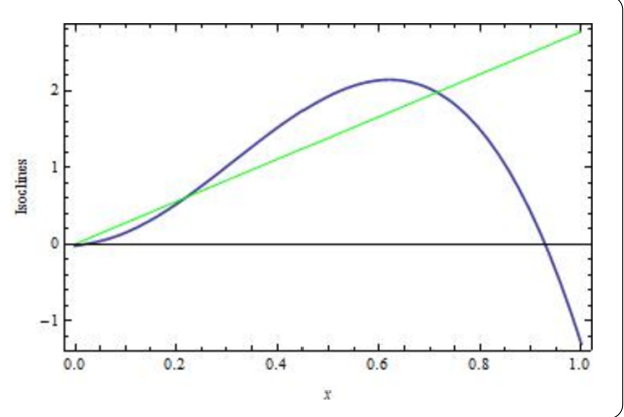

Figure 2 Existence of unique positive equilibrium for $\alpha=0.042, \beta=0.59, a=0.155, b=0.465, d=0.38$

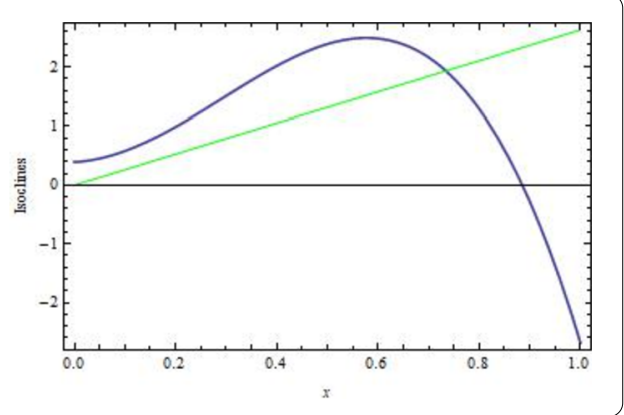

and

$$
w_{12}:=-\frac{\alpha h x^{*}}{\alpha \beta+x^{*}\left(x^{*}+\alpha\right)} .
$$

Furthermore, the characteristic equation for $J\left(x^{*}, y^{*}\right)$ is given as follows:

$$
P(\lambda)=\lambda^{2}-\left(1-c h+w_{11}\right) \lambda+w_{11}(1-c h)-\frac{w_{12} c h}{d} .
$$

Keeping in view the relation between roots and coefficients of a quadratic equation [26, 27], we have the following lemma for the dynamics of interior equilibrium $\left(x^{*}, y^{*}\right)$.

Lemma 2.1 The following hold true for interior equilibrium $\left(x^{*}, y^{*}\right)$ of system (2):

(i) $\left(x^{*}, y^{*}\right)$ is a sink if

$$
\begin{aligned}
& w_{11}+\frac{w_{12}}{d}<1, \\
& w_{12} \text { ch }<d\left(1+w_{11}\right)(2-c h),
\end{aligned}
$$

and

$$
w_{11}(1-c h)<1+\frac{w_{12} c h}{d}
$$

(ii) $\left(x^{*}, y^{*}\right)$ is a saddle point if

$$
1-w_{11}-\frac{w_{12}}{d}>0
$$


and

$$
\left(1+w_{11}\right)(2-c h)-\frac{w_{12} c h}{d}<0 .
$$

(iii) $\left(x^{*}, y^{*}\right)$ is a source (repeller) if

$$
\left|w_{11}(1-c h)-\frac{w_{12} c h}{d}\right|>1
$$

and

$$
\left|1-c h+w_{11}\right|<\left|1+w_{11}(1-c h)-\frac{w_{12} c h}{d}\right| .
$$

(iv) $\left(x^{*}, y^{*}\right)$ is a non-hyperbolic point if

$$
\left|1-c h+w_{11}\right|=\left|1+w_{11}(1-c h)-\frac{w_{12} c h}{d}\right|
$$

and

$$
\left|w_{11}(1-c h)-\frac{w_{12} c h}{d}\right| \neq 1
$$

or

$$
w_{11}(1-c h)-\frac{w_{12} c h}{d}=1 \quad \text { and } \quad\left|1-c h+w_{11}\right|<2 .
$$

\section{Period-doubling bifurcation}

In this section, we study the period-doubling bifurcation (PDB) at the fixed point $E^{*}=$ $\left(x^{*}, y^{*}\right)$ of system (2). The characteristic polynomial equation at $E^{*}$ has eigenvalues $\lambda_{1}=-1$ and $\left|\lambda_{2}\right| \neq 1$. The characteristic polynomial equation for system (2) at $E^{*}=\left(x^{*}, y^{*}\right)$ can be written as follows:

$$
P(\lambda)=\lambda^{2}-A \lambda+B
$$

where $A=\left(1-c h+w_{11}\right)$ and $B=w_{11}(1-c h)-\frac{w_{12} c h}{d}$.

Assume that $A^{2}>4 B$, we get

$$
\left(1-c h+w_{11}\right)^{2}>4 w_{11}(1-c h)-\frac{w_{12} c h}{d},
$$

and $A+B=-1$, we obtain

$$
h=\frac{2\left(1+w_{11}\right) d}{c\left(\left(1+w_{11}\right) d+w_{12}\right)} .
$$

Then the solutions of $P(\lambda)=0$ are $\lambda_{1}=-1$ and $\lambda_{2}=-B$. The condition $\left|\lambda_{2}\right| \neq 1$ implies that

$$
w_{11}(1-c h)-\frac{w_{12} c h}{d} \neq \pm 1 .
$$


Let

$$
\begin{aligned}
\Omega_{\mathrm{PD}}= & \left\{(a, b, c, d, h, \alpha, \beta): A^{2}>4 B, h=\frac{2\left(1+w_{11}\right) d}{c\left(\left(1+w_{11}\right) d+w_{12}\right)}, B \neq \pm 1, a, c, d, h, \alpha,\right. \\
& \beta>0,0<b<1\} .
\end{aligned}
$$

PDB occurs in system (2) when we change real parameters in the neighborhood of $\Omega_{\mathrm{PD}}$. Let

$$
h=\frac{2\left(1+w_{11}\right) d}{c\left(\left(1+w_{11}\right) d+w_{12}\right)}:=h_{1}
$$

and taking parameters $(a, b, c, d, h, \alpha, \beta) \in \Omega_{\mathrm{PD}}$ arbitrary, then in terms of parameters $(a, b, c, d, h, \alpha, \beta)$ model (2) can be expressed as

$$
\left(\begin{array}{l}
x \\
y
\end{array}\right) \rightarrow\left(\begin{array}{c}
x+h_{1}\left(x(1-x)-\frac{x y}{\frac{x^{2}}{\alpha}+x+\beta}-\frac{a x}{x+b}\right) \\
y+h_{1}\left(c y\left(1-\frac{d y}{x}\right)\right)
\end{array}\right) .
$$

Now, taking $\tilde{h}$ as a small bifurcation parameter, a perturbation map (6) is given as

$$
\left(\begin{array}{l}
x \\
y
\end{array}\right) \rightarrow\left(\begin{array}{c}
x+\left(h_{1}+\tilde{h}\right)\left(x(1-x)-\frac{x y}{\frac{x^{2}}{\alpha}+x+\beta}-\frac{a x}{x+b}\right) \\
y+\left(h_{1}+\tilde{h}\right)\left(c y\left(1-\frac{d y}{x}\right)\right)
\end{array}\right) .
$$

Now, we consider the transformation $u=x-x^{*}$ and $v=y-y^{*}$, then map (7) is transformed in the following fashion:

$$
\left(\begin{array}{l}
u \\
v
\end{array}\right) \rightarrow\left(\begin{array}{ll}
a_{11} & a_{12} \\
a_{21} & a_{22}
\end{array}\right)\left(\begin{array}{l}
u \\
v
\end{array}\right)+\left(\begin{array}{l}
f(u, v, \tilde{h}) \\
g(u, v, \tilde{h})
\end{array}\right),
$$

where

$$
\begin{aligned}
& f(u, v, \tilde{h})=a_{13} u^{2}+a_{14} u v+b_{1} u^{3}+b_{2} u^{2} v+b_{3} u^{2} \tilde{h}+e_{1} u v \tilde{h}+e_{2} u \tilde{h}+e_{3} v \tilde{h} \\
& +O\left((|u|+|v|)^{4}\right), \\
& g(u, v, \tilde{h})=a_{23} u^{2}+a_{24} v^{2}+a_{25} u v+k_{1} u v^{2}+k_{2} u^{3}+k_{3} u^{2} v+k_{4} v^{2} \tilde{h}+k_{5} u^{2} \tilde{h} \\
& +k_{6} u v \tilde{h}+k_{7} u \tilde{h}+k_{8} v \tilde{h}+O\left((|u|+|v|)^{4}\right), \\
& a_{11}=1+h_{1}\left(\frac{a x^{*}}{\left(b+x^{*}\right)^{2}}+\frac{x^{*} y^{*}\left(1+\frac{2 x^{*}}{\alpha}\right)}{\left(\beta+x^{*}\left(\frac{x^{*}}{\alpha}+1\right)\right)^{2}}-x^{*}\right), \quad a_{12}=-\frac{\alpha h_{1} x^{*}}{\alpha \beta+x^{*}\left(x^{*}+\alpha\right)}, \\
& a_{13}=h_{1}\left(-1+\frac{y^{*} \alpha\left(\alpha^{2} \beta+3 \alpha \beta x^{*}-x^{* 3}\right)}{\left(\left(\beta+x^{*}\right) \alpha+x^{* 2}\right)^{3}}+\frac{a b}{\left(b+x^{*}\right)^{3}}\right) \text {, } \\
& a_{14}=-h_{1} \alpha\left(\frac{\alpha \beta-x^{* 2}}{\left(\left(\beta+x^{*}\right) \alpha+x^{* 2}\right)^{2}}\right), \quad a_{21}=\frac{c h_{1}}{d}, \quad a_{22}=1-c h_{1}, \\
& a_{23}=-\left(\frac{h_{1} c y^{* 2} d}{x^{* 3}}\right), \quad a_{24}=-\left(\frac{h_{1} c d}{x^{*}}\right), \quad a_{25}=\left(\frac{2 h_{1} c y^{*} d}{x^{* 2}}\right),
\end{aligned}
$$




$$
\begin{aligned}
& b_{1}=h_{1}\left(\frac{y^{*} \alpha\left(-\beta \alpha^{3}+\beta\left(\beta-4 x^{*}\right) \alpha^{2}-6 \alpha \beta x^{* 2}+x^{* 4}\right)}{\left(\left(\beta+x^{*}\right) \alpha+x^{* 2}\right)^{4}}-\frac{a b}{\left(b+x^{*}\right)^{4}}\right), \\
& b_{2}=h_{1} \alpha\left(\frac{\left(\alpha^{2} \beta+3 \alpha \beta x^{*}-x^{* 3}\right)}{\left(\left(\beta+x^{*}\right) \alpha+x^{* 2}\right)^{3}}, \quad b_{3}=\left(-1+\frac{y^{*} \alpha\left(\alpha^{2} \beta+3 \alpha \beta x^{*}-x^{* 3}\right.}{\left(\left(\beta+x^{*}\right) \alpha+x^{* 2}\right)^{3}}+\frac{a b}{\left(b+x^{*}\right)^{3}}\right),\right. \\
& e_{1}=\left(\frac{-\alpha\left(\alpha \beta-x^{* 2}\right)}{\left(\left(\beta+x^{*}\right) \alpha+x^{* 2}\right)^{2}}\right), \quad e_{2}=-2 x^{*}+1-\frac{y^{*} \alpha\left(\alpha \beta-x^{* 2}\right)}{\left(\left(\beta+x^{*}\right) \alpha+x^{* 2}\right)^{2}}-\frac{a b}{\left(b+x^{*}\right)^{2}}, \\
& e_{3}=-\frac{\alpha x^{*}}{\left(\beta+x^{*}\right) \alpha+x^{* 2}}, \quad k_{1}=\left(\frac{h_{1} c d}{x^{* 2}}\right), \quad k_{2}=\left(\frac{h_{1} c y^{* 2} d}{x^{* 4}}\right), \\
& k_{3}=-\left(\frac{2 h_{1} c d y^{*}}{x^{* 3}}\right), \quad k_{4}=-\left(\frac{c d}{x^{*}}\right), \quad k_{5}=-\left(\frac{c d y^{* 2}}{x^{* 3}}\right), \\
& k_{6}=\left(\frac{2 c d y^{*}}{x^{* 2}}\right), \quad k_{7}=\left(\frac{c d y^{* 2}}{x^{* 2}}\right), \quad k_{8}=-c\left(\frac{2 d y^{*}-x^{*}}{x^{*}}\right) .
\end{aligned}
$$

We diagonalize system (8) by considering the following translation:

$$
\left(\begin{array}{l}
u \\
v
\end{array}\right)=T\left(\begin{array}{l}
p \\
q
\end{array}\right)
$$

where $T=\left(\begin{array}{cc}a_{12} & a_{12} \\ -1-a_{11} & \lambda_{2}-a_{11}\end{array}\right)$ and $|T| \neq 0$. Using translation (9), map (8) can be expressed as

$$
(p) \rightarrow\left(\begin{array}{cc}
-1 & 0 \\
0 & \lambda_{2}
\end{array}\right)\left(\begin{array}{l}
p \\
q
\end{array}\right)+\left(\begin{array}{l}
\tilde{f}(p, q, \tilde{h}) \\
\tilde{g}(p, q, \tilde{h})
\end{array}\right)
$$

where

$$
\begin{aligned}
\tilde{f}(p, q, \tilde{h})= & \left(\chi a_{13}-\frac{a_{23}}{\lambda_{2}+1}\right) u^{2}+\left(b_{1} \chi-\frac{k_{2}}{\lambda_{2}+1}\right) u^{3}+\left(b_{2} \chi-\frac{k_{3}}{\lambda_{2}+1}\right) u^{2} v \\
& +\left(a_{14} \chi-\frac{a_{25}}{\lambda_{2}+1}\right) u v+\left(b_{3} \chi-\frac{k_{5}}{\lambda_{2}+1}\right) \tilde{h} u^{2}+\left(e_{1} \chi-\frac{k_{6}}{\lambda_{2}+1}\right) \tilde{h} u v \\
& +\left(e_{2} \chi-\frac{k_{7}}{\lambda_{2}+1}\right) \tilde{h} u+\left(e_{3} \chi-\frac{k_{8}}{\lambda_{2}+1}\right) \tilde{h} v+\left(\frac{-k_{1}}{\lambda_{2}+1}\right) u v^{2} \\
& +\left(\frac{-k_{4}}{\lambda_{2}+1}\right) \tilde{h} v^{2}+\left(-\frac{a_{24}}{\lambda_{2}+1}\right) v^{2}, \\
\tilde{g}(p, q, \tilde{h})= & \left(\theta a_{13}+\frac{a_{23}}{\lambda_{2}+1}\right) u^{2}+\left(\theta b_{1}+\frac{k_{2}}{\lambda_{2}+1}\right) u^{3}+\left(\theta b_{2}+\frac{k_{3}}{\lambda_{2}+1}\right) u^{2} v \\
& +\left(\theta a_{14}+\frac{a_{25}}{\lambda_{2}+1}\right) u v+\left(\theta b_{3}+\frac{k_{5}}{\lambda_{2}+1}\right) \tilde{h} u^{2}+\left(\theta e_{1}+\frac{k_{6}}{\lambda_{2}+1}\right) \tilde{h} u v \\
& +\left(\theta e_{2}+\frac{k_{7}}{\lambda_{2}+1}\right) \tilde{h} u+\left(\theta e_{3}+\frac{k_{8}}{\lambda_{2}+1}\right) \tilde{h} v+\left(\frac{k_{1}}{\lambda_{2}+1}\right) u v^{2} \\
& +\left(\frac{k_{4}}{\lambda_{2}+1}\right) \tilde{h} v^{2}+\left(\frac{a_{24}}{\lambda_{2}+1}\right) v^{2},
\end{aligned}
$$

$\chi=\left(\frac{\left(\lambda_{2}-a_{11}\right)}{a_{12}\left(\lambda_{2}+1\right)}\right), \theta=\left(\frac{\left(1+a_{11}\right)}{a_{12}\left(\lambda_{2}+1\right)}\right), u=a_{12}(p+q)$ and $v=-\left(1+a_{11}\right) p+\left(\lambda_{2}-a_{11}\right) q$. Now, we approximate center manifold $(C M)$ at the origin by using the center manifold theorem (CMT) for system (10) depending on parameter $\tilde{h}$. The dynamics of map (8) around the 
fixed point $(0,0)$ for parameter values near $\tilde{h}=0$ can be analyzed using the behavior of a one-parameter family of maps on the CM, according to CMT. Let $G^{c}$ be the CM of (10) calculated at origin, then $G^{c}$ can be written as follows:

$$
G^{c}(0,0,0)=\left\{(p, q, \tilde{h}) \in R^{3}: q=z_{2}(p, \tilde{h}), z_{2}(0,0)=D z_{2}(0,0)=0\right\} .
$$

The two conditions $z_{2}(0,0)=0$ and $D z_{2}(0,0)=0$ show that the CM is tangent to the $q=0$ axis. Assume that $z_{2}(p, \tilde{h})$ has the following form:

$$
z_{2}(p, \tilde{h})=m_{1} p^{2}+m_{2} p \tilde{h}+m_{3} \tilde{h}^{2}+O\left((|p|+|\tilde{h}|)^{3}\right) .
$$

The following equation must be satisfied by CM of (11):

$$
X\left(z_{2}(p, \tilde{h})\right)=z_{2}\left(-p+\tilde{f}\left(p, z_{2}(p, \tilde{h}), \tilde{h}\right)\right)-\lambda_{2} z_{2}(p, \tilde{h})-\tilde{g}\left(p, z_{2}(p, \tilde{h}), \tilde{h}\right)=0 .
$$

Substituting (10), (11) into (12) and comparing the coefficients, we obtain

$$
\begin{aligned}
m_{1}= & \frac{1}{-\lambda_{2}+1}\left(\left(\theta a_{13}+\frac{a_{23}}{\lambda_{2}+1}\right) a_{12}^{2}+\left(a_{14} \theta+\frac{a_{25}}{\lambda_{2}+1}\right) a_{12}\left(-1-a_{11}\right)\right. \\
& \left.+a_{24}\left(\frac{\left(-1-a_{11}\right)^{2}}{\lambda_{2}+1}\right)\right), \\
m_{2}= & \frac{-1}{\lambda_{2}+1}\left(\left(e_{2} \theta+\frac{k_{7}}{\lambda_{2}+1}\right) a_{12}+\left(e_{3} \theta+\frac{k_{8}}{\lambda_{2}+1}\right)\left(-1-a_{11}\right)\right), \\
m_{3}= & 0 .
\end{aligned}
$$

Then the confined one-dimensional map to the $\mathrm{CM} \mathrm{G}^{c}$ becomes

$$
F: p \longrightarrow-p+s_{1} p^{2}+s_{2} p \tilde{h}+s_{3} p^{2} \tilde{h}+s_{4} p \tilde{h}^{2}+s_{5} p^{3}+O\left((|p|+|\tilde{h}|)^{4}\right)
$$

where

$$
\begin{aligned}
s_{1}= & \left(\left(\theta a_{13}+\frac{a_{23}}{\lambda_{2}+1}\right) a_{12}^{2}+\left(\theta a_{14}+\frac{a_{25}}{\lambda_{2}+1}\right) a_{12}\left(-1-a_{11}\right)+a_{24}\left(\frac{\left(-1-a_{11}\right)^{2}}{\lambda_{2}+1}\right)\right), \\
s_{2}= & \left(\left(\theta e_{2}+\frac{k_{7}}{\lambda_{2}+1}\right) a_{12}+\left(\theta e_{3}+\frac{x k_{6}+k_{8}}{\lambda_{2}+1}\right)\left(-1-a_{11}\right)\right), \\
s_{3}= & \left(2\left(\theta a_{13}+\frac{a_{23}}{\lambda_{2}+1}\right) a_{12}^{2} m_{2}+\left(\theta b_{3}+\frac{k_{5}}{\lambda_{2}+1}\right) a_{12}^{2}+\left(\theta a_{14}+\frac{a_{25}}{\lambda_{2}+1}\right) a_{12} m_{2}\left(\lambda_{2}\right.\right. \\
& \left.-2 a_{11}-1\right)+\left(\theta e_{1} a_{12}\right)\left(-1-a_{11}\right)+\left(e_{2} \theta+\frac{k_{7}}{\lambda_{2}+1}\right) a_{12} m_{1}+\left(2 a_{12} a_{24} \chi(-1\right. \\
& \left.\left.\left.-a_{11}\right) m_{2}\right)+k_{4}\left(\left(1+a_{11}\right) \theta a_{12}\right)+\left(\theta e_{3}+\frac{x k_{6}+k_{8}}{\lambda_{2}+1}\right)\left(\lambda_{2}-a_{11}\right) m_{1}\right), \\
s_{4}= & \left(2\left(\theta a_{13}+\frac{a_{23}}{\lambda_{2}+1}\right) a_{12}^{2} m_{3}+\left(\theta a_{14}+\frac{a_{25}}{\lambda_{2}+1}\right) a_{12} m_{3}\left(\lambda_{2}-2 a_{11}-1\right)+\left(e_{2} \theta\right.\right. \\
& \left.\left.+\frac{k_{7}}{\lambda_{2}+1}\right) a_{12} m_{2}+\left(2 a_{12} a_{24} \chi\left(-1-a_{11}\right) m_{3}\right)+\left(\theta e_{3}+\frac{x k_{6}+k_{8}}{\lambda_{2}+1}\right)\left(\lambda_{2}-a_{11}\right) m_{2}\right),
\end{aligned}
$$




$$
\begin{aligned}
s_{5}= & \left(\left(\theta b_{1}+\frac{k_{2}}{\lambda_{2}+1}\right) a_{12}^{3}+\left(\theta b_{2}+\frac{k_{3}}{\lambda_{2}+1}\right) a_{12}^{2}\left(-1-a_{11}\right)+2\left(\theta a_{13}+\frac{a_{23}}{\lambda_{2}+1}\right) a_{12}^{2} m_{1}\right. \\
& +k_{1} a_{12}^{2} \theta\left(1+a_{11}\right)+\left(\theta a_{14}+\frac{a_{25}}{\lambda_{2}+1}\right) a_{12} m_{1}\left(\lambda_{2}-2 a_{11}-1\right) \\
& \left.+\left(2 a_{12} a_{24} \chi\left(-1-a_{11}\right) m_{1}\right)\right) .
\end{aligned}
$$

If map (13) undergoes a period-doubling bifurcation, then we need that two discriminatory quantities $l_{1}$ and $l_{2}$ are not zero at $(p, \tilde{h})=(0,0)$ :

$$
\begin{aligned}
& l_{1}=\left(\frac{\partial^{2} F}{\partial p \partial \tilde{h}}+\frac{1}{2} \frac{\partial F}{\partial \tilde{h}} \frac{\partial^{2} F}{\partial p^{2}}\right)_{(0,0)}=s_{2} \neq 0, \\
& l_{2}=\left(\frac{1}{6} \frac{\partial^{3} F}{\partial p^{3}}+\frac{1}{2}\left(\frac{\partial^{2} F}{\partial p^{2}}\right)^{2}\right)_{(0,0)}=s_{1}^{2}+s_{5} \neq 0 .
\end{aligned}
$$

Theorem 3.1 If $l_{1} \neq 0$ and $l_{2} \neq 0$, then model (2) undergoes PDB at $E^{*}=\left(x^{*}, y^{*}\right)$ while we change the real parameter $h$ nearby $h_{1}$. Moreover, if $l_{2}>0$, then the period-two orbits that bifurcate from positive steady-state are stable, and if $l_{2}<0$, then these orbits are unstable.

\section{Neimark-Sacker bifurcation}

In this section, we study the existence of Neimark-Sacker bifurcation (NSB) for the steady state $\left(x^{*}, y^{*}\right)$ of system (2). We find the parametric conditions for a non-hyperbolic fixed point so that the Jacobian matrix has two complex conjugate eigenvalues with modulus 1 . At fixed point $E^{*}=\left(x^{*}, y^{*}\right)$, the characteristic polynomial equation can be written as

$$
P(\lambda)=\lambda^{2}-A \lambda+B
$$

where

$$
A=\left(1-c h+w_{11}\right) \quad \text { and } \quad B=w_{11}(1-c h)-\frac{w_{12} c h}{d} .
$$

From Lemma 2.1, the solutions of $P(\lambda)=0$ are complex conjugates with modulus 1 if $B=1$ and $|A|<2$, which implies that $h_{2}=\left(\frac{d\left(w_{11}-1\right)}{c\left(d w_{11}+w_{12}\right)}\right),\left|1-c h+w_{11}\right|<2$.

Assume that

$$
\Omega_{\mathrm{NS}}=\left\{\left(a, b, c, d, h_{2}, \alpha, \beta\right): h_{2}=\frac{d\left(w_{11}-1\right)}{c\left(d w_{11}+w_{12}\right)},\left|1-c h_{2}+w_{11}\right|<2,(a, b, c, d, \alpha, \beta)>0\right\} .
$$

When the parameters are changing in a small neighborhood of $\Omega_{\mathrm{NS}}$, the unique positive equilibrium point of system (2) experiences the Neimark-Sacker bifurcation. Choosing the parameters $\left(h_{2}, a, b, c, d, \alpha, \beta\right)$ in a random way from the set $\Omega_{\mathrm{NS}}$, system (2) can be described by the following map:

$$
\left(\begin{array}{l}
x \\
y
\end{array}\right) \rightarrow\left(\begin{array}{c}
x+h_{2}\left(x(1-x)-\frac{x y}{\frac{x^{2}}{\alpha}+x+\beta}-\frac{a x}{x+b}\right) \\
y+h_{2}\left(c y\left(1-\frac{d y}{x}\right)\right)
\end{array}\right)
$$


Since $\left(h_{2}, a, b, c, d, \alpha, \beta\right) \in \Omega_{\mathrm{NS}}$, where

$$
h_{2}=\left(\frac{d\left(w_{11}-1\right)}{c\left(d w_{11}+w_{12}\right)}\right) \text {. }
$$

Take $\tilde{h}$ as a bifurcation parameter and consider system (15) as follows:

$$
\left(\begin{array}{l}
x \\
y
\end{array}\right) \longrightarrow\left(\begin{array}{c}
x+\left(h_{2}+\tilde{h}\right)\left(x(1-x)-\frac{x y}{\frac{x^{2}}{\alpha}+x+\beta}-\frac{a x}{x+b}\right) \\
y+\left(h_{2}+\tilde{h}\right)\left(c y\left(1-\frac{d y}{x}\right)\right)
\end{array}\right),
$$

where $|\tilde{h}|<<<1$, which is a small perturbation parameter. Next we consider the transformation $u=x-x^{*}, v=y-y^{*}$, then map (16) is transferred into the following form:

$$
\left(\begin{array}{l}
u \\
v
\end{array}\right) \rightarrow\left(\begin{array}{ll}
a_{11} & a_{12} \\
a_{21} & a_{22}
\end{array}\right)\left(\begin{array}{l}
u \\
v
\end{array}\right)+\left(\begin{array}{l}
f(u, v) \\
g(u, v)
\end{array}\right),
$$

where

$$
\begin{aligned}
& f(u, v)=a_{13} u^{2}+a_{14} u v+b_{1} u^{3}+b_{2} u^{2} v+O\left((|u|+|v|)^{4}\right), \\
& g(u, v)=a_{23} u^{2}+a_{24} v^{2}+a_{25} u v+k_{1} u v^{2}+k_{2} u^{3}+k_{3} u^{2} v+O\left((|u|+|v|)^{4}\right) .
\end{aligned}
$$

All the coefficients are given in (8) by replacing $h_{1}=h_{2}+\tilde{h}$. The characteristic polynomial equation of linearized system (17) evaluated at $(0,0)$ is given by

$$
P(\lambda)=\lambda^{2}-A(\tilde{h}) \lambda+B(\tilde{h})=0
$$

$A(\tilde{h})=\left(1-c\left(h_{2}+\tilde{h}\right)+w_{11}\right)$, and $B(\tilde{h})=w_{11}\left(1-c\left(h_{2}+\tilde{h}\right)\right)-\frac{w_{12} c\left(h_{2}+\tilde{h}\right)}{d}$. Since parameter $\left(h_{2}, a, b, c, d, \alpha, \beta\right) \in \Omega_{\mathrm{NS}}$, therefore the roots of (18) are complex numbers $\lambda_{1}, \lambda_{2}$ with $\lambda_{2}=\overline{\lambda_{1}}$ and $\left|\lambda_{1}\right|=\left|\lambda_{2}\right|=1$, it follows that

$$
\lambda_{1}, \lambda_{2}=\frac{A(\tilde{h})}{2} \pm \frac{\iota}{2} \sqrt{4 B(\tilde{h})-A^{2}(\tilde{h})} .
$$

Then we obtain

$$
\begin{aligned}
& \left|\lambda_{1}\right|=\left|\lambda_{2}\right|=\sqrt{B(\tilde{h}),} \\
& \left(\frac{d\left|\lambda_{2}\right|}{d \tilde{h}}\right)_{\tilde{h}=0}=\left(\frac{d\left|\lambda_{1}\right|}{d \tilde{h}}\right)_{\tilde{h}=0}=\frac{1}{2}\left(\frac{c\left(d w_{11}-w_{12}\right)}{d \sqrt{\frac{\left(-d-2 w_{12}\right) w_{11}+w_{12}}{d w_{11}+w_{12}}}}\right) \neq 0 .
\end{aligned}
$$

Further, we need that when $\tilde{h}=0, \lambda_{1}^{m}, \lambda_{2}^{m} \neq 1$ for $m=1,2,3,4$, which is equivalent to $A(0) \neq$ $2,1,0,-2$. Since $\left(h_{2}, a, b, c, d, \alpha, \beta\right) \in \Omega_{\mathrm{NS}}$, it follows that

$$
-2<A(0)=\left(1-c h+w_{11}\right)<2 .
$$

Next we assume that $A(0) \neq 0,1$, that is,

$$
w_{11}-c h \neq-1 \text { and } c h \neq w_{11} \text {. }
$$


Thus, we obtain $A(0) \neq 2,1,0,-2$, which gives $\lambda_{1}^{m}, \lambda_{2}^{m} \neq 1 \forall m=1,2,3,4$ at $\tilde{h}=0$. Hence, $\tilde{h}=0$ roots of (18) do not lie in the intersection of unit circle of co-ordinate axis when $\tilde{h}=0$ and (19) hold. In order to obtain the normal form of (17) at $\tilde{h}=0$, we take $\alpha=\frac{A(0)}{2}$ and $\beta=\frac{1}{2} \sqrt{4 B(0)-A^{2}(0)}$. We take the transformation given as

$$
\left(\begin{array}{l}
u \\
v
\end{array}\right)=T\left(\begin{array}{l}
p \\
q
\end{array}\right)
$$

where $T=\left(\begin{array}{cc}a_{12} & 0 \\ \alpha-a_{11} & -\beta\end{array}\right)$ and $\operatorname{det}(T) \neq 0$. Under translation (20) the normal form of map (17) can be written as follows:

$$
\left(\begin{array}{l}
p \\
q
\end{array}\right) \rightarrow\left(\begin{array}{cc}
\alpha & -\beta \\
\beta & \alpha
\end{array}\right)\left(\begin{array}{l}
p \\
q
\end{array}\right)+\left(\begin{array}{l}
\tilde{f}(p, q) \\
\tilde{g}(p, q)
\end{array}\right)
$$

where

$$
\begin{aligned}
\tilde{f}(p, q)= & \frac{a_{13}}{a_{12}} u^{2}+\frac{a_{14}}{a_{12}} u v+\frac{b_{1}}{a_{12}} u^{3}+\frac{b_{2}}{a_{12}} u^{2} v+O\left((|p|+|q|)^{4}\right), \\
\tilde{g}(p, q)= & \left.\frac{\left(\alpha-a_{11}\right) a_{13}}{a_{12} \beta}-\frac{a_{23}}{\beta}\right) u^{2}+\left(\frac{\left(\alpha-a_{11}\right) a_{14}}{a_{12} \beta}-\frac{a_{25}}{\beta}\right) u v+\left(\frac{\left(\alpha-a_{11}\right) b_{2}}{a_{12} \beta}\right. \\
& \left.-\frac{k_{3}}{\beta}\right) u^{2} v-\frac{k_{1}}{\beta} u v^{2}-\frac{a_{24}}{\beta} v^{2}+\left(\frac{\left(\alpha-a_{11}\right) b_{1}}{a_{12} \beta}+\frac{k_{2}}{\beta}\right) u^{3}+O\left((|p|+|q|)^{4}\right),
\end{aligned}
$$

where $u=a_{12} p$ and $v=\left(\alpha-a_{11}\right) p-\beta q$. Next, we define the following nonzero real number:

$$
\begin{aligned}
& L=\left(\left[-\operatorname{Re}\left(\frac{\left(1-2 \lambda_{1}\right) \lambda_{2}^{2}}{1-\lambda_{1}} \zeta_{20} \zeta_{11}\right)-\frac{1}{2}\left|\zeta_{11}\right|^{2}-\left|\zeta_{02}\right|^{2}+\operatorname{Re}\left(\lambda_{2} \zeta_{21}\right)\right]\right)_{\tilde{h}=0}, \\
& \zeta_{20}=\frac{1}{8}\left[\tilde{f_{p p}}-\tilde{f}_{q q}+2 \tilde{g}_{p q}+\iota\left(\tilde{g}_{p p}-\tilde{g}_{q q}-2 \tilde{f}_{p q}\right)\right], \\
& \zeta_{11}=\frac{1}{4}\left[\tilde{f_{p p}}+\tilde{f}_{q q}+\iota\left(\tilde{g}_{p p}+\tilde{g}_{q q}\right)\right], \\
& \zeta_{02}=\frac{1}{8}\left[\tilde{f_{p p}}-\tilde{f}_{q q}-2 \tilde{g}_{p q}+\iota\left(\tilde{g}_{p p}-\tilde{g}_{q q}+2 \tilde{f}_{p q}\right)\right], \\
& \zeta_{21}=\frac{1}{16}\left[\tilde{f}_{p p p}+\tilde{f}_{p q q}+\tilde{g}_{p p q}+\tilde{g}_{q q q}+\iota\left(\tilde{g}_{p p p}+\tilde{g}_{p q q}-\tilde{f}_{p p q}-\tilde{f}_{q q q}\right)\right] .
\end{aligned}
$$

Due to the above analysis, we have the following result.

Theorem 4.1 If condition (19) holds and $L \neq 0$, then system (2) undergoes NSB at the unique positive equilibrium point $\left(x^{*}, y^{*}\right)$ when the parameter $h$ varies in a small neighborhood of $h_{2}=\left(\frac{d\left(w_{11}-1\right)}{c\left(d w_{11}+w_{12}\right)}\right)$. Furthermore, if $L>0$, then a repelling invariant closed curve bifurcates from the equilibrium point for $h<h_{2}$, which is also called sub-critical NSB, and an attracting invariant closed curve bifurcates when $L<0$, which is also called supercritical NSB.

\section{Chaos control}

In this segment, two feedback control techniques are talked about. The first one is the pole-placement methodology, which may be processed as a generalized OGY method, 
and the second method is known as the hybrid control strategy. In order to use the OGY technique to system (2), we have a tendency to write the system in the following way:

$$
\begin{aligned}
& x_{n+1}=x_{n}+h\left[x_{n}\left(1-x_{n}\right)-\frac{x_{n} y_{n}}{\frac{x_{n}^{2}}{\alpha}+x_{n}+\beta}-\frac{a x_{n}}{x_{n}+b}\right]=f\left(x_{n}, y_{n}, h\right), \\
& y_{n+1}=y_{n}+h\left[c y_{n}\left(1-d \frac{y_{n}}{x_{n}}\right)\right]=g\left(x_{n}, y_{n}, h\right),
\end{aligned}
$$

where $h$ indicates the parameter for chaos control. Furthermore, $h$ is limited to lie in some small interval $\left|h-h_{0}\right|<\epsilon$ with $\epsilon>0$, and $h_{0}$ indicates the nominal value belonging to the chaotic region. Suppose that $\left(x^{*}, y^{*}\right)$ indicates an unstable fixed point for (2) in the chaotic region, which yields under the influence of period-doubling and Neimark-Sacker bifurcation. In this case, (22) is approximated in the neighborhood of $\left(x^{*}, y^{*}\right)$ as follows:

$$
\left[\begin{array}{l}
x_{n+1}-x^{*} \\
y_{n+1}-y^{*}
\end{array}\right] \approx A\left[\begin{array}{l}
x_{n+1}-x^{*} \\
y_{n+1}-y^{*}
\end{array}\right]+B\left[\begin{array}{ll}
h & h_{0}
\end{array}\right]
$$

where

$$
A=\left[\begin{array}{ll}
\frac{\partial f}{\partial x_{n}}\left(x^{*}, y^{*}, h_{0}\right) & \frac{\partial f}{\partial y_{n}}\left(x^{*}, y^{*}, h_{0}\right) \\
\frac{\partial g}{\partial x_{n}}\left(x^{*}, y^{*}, h_{0}\right) & \frac{\partial g}{\partial y_{n}}\left(x^{*}, y^{*}, h_{0}\right)
\end{array}\right]=\left[\begin{array}{cc}
w_{11} & w_{12} \\
\frac{c h_{0}}{d} & 1-c h_{0}
\end{array}\right],
$$

and

$$
B=\left[\begin{array}{l}
\frac{\partial f}{\partial h}\left(x^{*}, y^{*}, h_{0}\right) \\
\frac{\partial g}{\partial h}\left(x^{*}, y^{*}, h_{0}\right)
\end{array}\right]=\left[\begin{array}{c}
w_{13} \\
0
\end{array}\right],
$$

where $w_{13}=x^{*}\left(1-x^{*}\right)-\frac{x^{* 2} \alpha}{\left(\left(x^{*}+\beta\right) \alpha+x^{* 2}\right) d}-\frac{a x^{*}}{\left(x^{*}+b\right)}$. Moreover, if the following matrix has rank 2 , then system (22) is controllable.

$$
C=[B: A B]=\left[\begin{array}{cc}
w_{13} & w_{11} w_{13} \\
0 & \frac{c h}{d} w_{13}
\end{array}\right] \text {. }
$$

Since $\frac{c h}{d} w_{13} \neq 0$, the rank of $C$ is 2 . Consequently, system (2) is always controllable with the OGY feedback control method. Next we assume that $\left[h-h_{o}\right]=-K\left[\begin{array}{l}x_{n}-x^{*} \\ y_{n}-y^{*}\end{array}\right]$, where $K=$ $\left[k_{1} k_{2}\right]$, then system (23) can be written as

$$
\left[\begin{array}{c}
x_{n+1}-x^{*} \\
y_{n+1}-y^{*}
\end{array}\right] \approx[A-B K]\left[\begin{array}{l}
x_{n}-x^{*} \\
y_{n}-y^{*}
\end{array}\right] .
$$

Moreover, both eigenvalues $\mu_{1}$ and $\mu_{2}$ of matrix $A-B K$ lie in an open disk if and only if the equilibrium point $\left(x^{*}, y^{*}\right)$ is locally asymptotically stable. These eigenvalues are called regulator poles, and the problem of placing these regulator poles at appropriate place is known as a pole-placement problem. Since the rank of matrix $C$ is 2 , hence the poleplacement problem has a unique solution. Next we suppose that $\gamma^{2}+\alpha_{1} \gamma+\alpha_{2}$ is the characteristic equation of $\mathrm{A}$ and $\mu^{2}+\beta_{1} \mu+\beta_{2}$ is the characteristic equation of $A-B K$, then 
the unique solution of pole-placement problem is given by

$$
K=\left[\begin{array}{ll}
\beta_{2}-\alpha_{2} & \beta_{1}-\alpha_{1}
\end{array}\right] T^{-1},
$$

where $T=C M$ and $M=\left[\begin{array}{cc}\alpha_{1} & 1 \\ 1 & 0\end{array}\right]$.

Secondly, we apply the hybrid control strategy to system (2) for controlling chaos through the effect of both types of bifurcations. Assume that system (2) undergoes PDB and NSB at its equilibrium point $\left(x^{*}, y^{*}\right)$, then the corresponding controlled system using the hybrid control method is written as follows:

$$
\begin{aligned}
& x_{n+1}=\rho\left[x_{n}+h\left(x_{n}\left(1-x_{n}\right)-\frac{x_{n} y_{n}}{\frac{x_{n}^{2}}{\alpha}+x_{n}+\beta}-\frac{a x_{n}}{x_{n}+b}\right)\right]+(1-\rho) x_{n}, \\
& y_{n+1}=\rho\left[y_{n}+h\left(c y_{n}\left(1-d \frac{y_{n}}{x_{n}}\right)\right]+(1-\rho) y_{n},\right.
\end{aligned}
$$

where $\rho \in(0,1)$ illustrates the control parameter for controlled system (26). Moreover, the Jacobian matrix for $(26)$ at $\left(x^{*}, y^{*}\right)$ is computed as follows:

$$
J=\left[\begin{array}{cc}
\rho w_{11}+(1-\rho) & \rho w_{12} \\
\rho \frac{c h}{d} & \rho(1-c h)+(1-\rho)
\end{array}\right] .
$$

Furthermore, the following lemma gives parametric conditions for local stability of the fixed point $\left(x^{*}, y^{*}\right)$ for controlled system (26).

Lemma 5.1 The positive equilibrium point $\left(x^{*}, y^{*}\right)$ of control system (26) is locally asymptotically stable if the following condition holds:

$$
d<\frac{\operatorname{ch} \rho^{2} w_{12}}{\left(1-\operatorname{ch} \rho\left(\rho w_{11}-\rho+1\right)+\rho\left(w_{11}-1\right)\right)} .
$$

\section{Numerical simulation and discussion}

This segment is committed to proving the presence of PDB and NSB for system (2) for specific numerical values of its parameters $(a, b, c, d, \alpha, \beta)$ whereas taking $h$ as bifurcation parameter. The verification of period-doubling and Neimark-Sacker bifurcation is demonstrated using bifurcation diagrams and phase portraits. In addition, hybrid control and pole-placement techniques for chaos control are too supported by numerical simulations.

Example 6.1 First we choose $(a, b, c, d, \alpha, \beta)=(0.4,2,5.4,0.113,0.012,6)$ and the initial values $\left(x_{0}, y_{0}\right)=(0.75,6.5)$. Then system (2) experiences PDB as bifurcation parameter $h$ changes in the small neighborhood of $h_{0}=0.3805$. From bifurcation diagrams, which are shown in Fig. 3, for $h<0.3805$, the fixed point $E^{*}$ is stable and unstable at $h=0.3805$ and period-doubling bifurcation occurs for $\mathrm{h}>0.3805$. MLEs related to bifurcation diagrams Fig. 3(a) and (b) are plotted in Fig. 3(c), which confirms the existence of chaotic behavior in system (2). The phase portraits related to Figs. 3(a) and (b) are plotted in Fig. 4. On the other hand, for these parametric values $\lambda_{1}=-1, \lambda_{2}=0.7604011396 \neq \pm 1$, $l_{1}=-11.13714613 \neq 0$, and $l_{2}=0.3269262762 \neq 0$. Therefore, all the conditions of Theorem 3.1 are satisfied. 


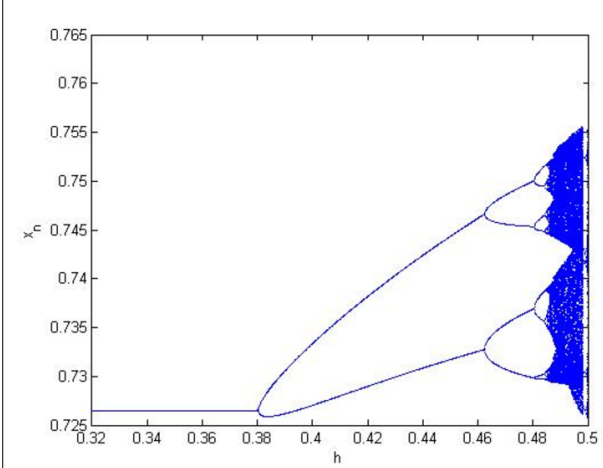

(a) Bifurcation diagram of $x_{n}$

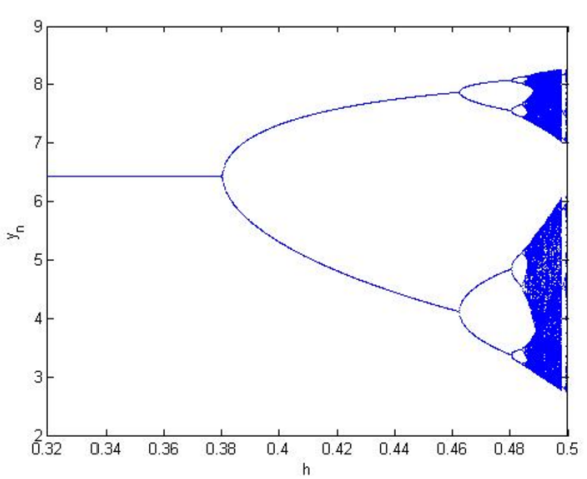

(b) Bifurcation diagram of $y_{n}$

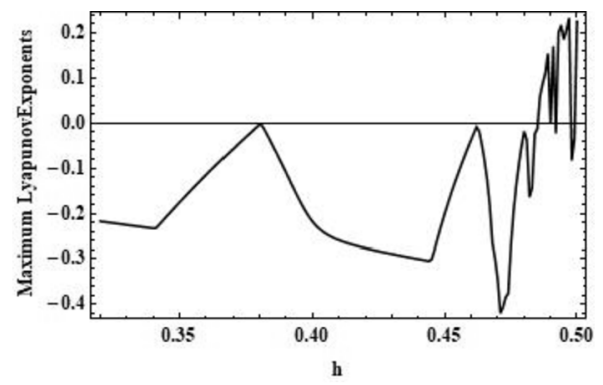

(c) Maximum Lyapunov exponents

Figure 3 MLE and bifurcation diagrams for model (2) in the interval $0.32 \leq h \leq 0.5$ with parameters $(a, b, c, d, \alpha, \beta, h)=(0.4,2,5.4,0.113,0.012,6,0.3805)$ and initial conditions $\left(x_{0}, y_{0}\right)=(0.75,6.5)$

Example 6.2 Next we select $(a, b, c, d, \alpha, \beta)=(0.001,0.75,0.224,0.29,0.19,1)$ and $h \in$ $[0.4,0.99]$ and the initial value $\left(x_{0}, y_{0}\right)=(0.38564,1.33674)$. Then system (2) undergoes NSB as bifurcation parameter $h_{0}=0.5731$. From bifurcation diagrams $5(\mathrm{a})$ and (b), it can be easily seen that $E^{*}$ of map (2) is stable for $h<0.5731$ and unstable at $h=0.5731$, and NSB occurs at $h>0.5731$. MLEs related to bifurcation diagrams Figs. 5(a) and (b) are plotted in Fig. 5(c), which confirms the existence of chaotic behavior in system (2). Phase portraits related to these parametric values are shown in Fig. 6. For these parametric values, the first Lyapunov exponent is computed as follows: $L=-0.03295317044 \neq 0$. This proves the correctness of Theorem 4.1.

Example 6.3 For an application of the pole-placement control strategy for NeimarkSacker bifurcation to system (2), we select the nominal value $h_{0}=0.575$ that belongs to the chaotic region $[0.5731,0.99]$. At $(a, b, c, d, \alpha, \beta)=(0.001,0.75,0.224,0.29,0.19,1)$ the unique positive equilibrium point $\left(x^{*}, y^{*}\right)=(0.384976,1.33584)$ of system $(2)$ and it is unstable. In this case system (22) can be written as

$$
\begin{aligned}
& x_{n+1}=x_{n}+h\left[x_{n}\left(1-x_{n}\right)-\frac{x_{n} y_{n}}{\frac{x_{n}^{2}}{0.19}+x_{n}+1}-\frac{0.001 x_{n}}{x_{n}+0.75}\right]=f\left(x_{n}, y_{n}, h\right), \\
& y_{n+1}=y_{n}+h\left[0.224 y_{n}\left(1-0.29 \frac{y_{n}}{x_{n}}\right)\right]=g\left(x_{n}, y_{n}, h\right) .
\end{aligned}
$$




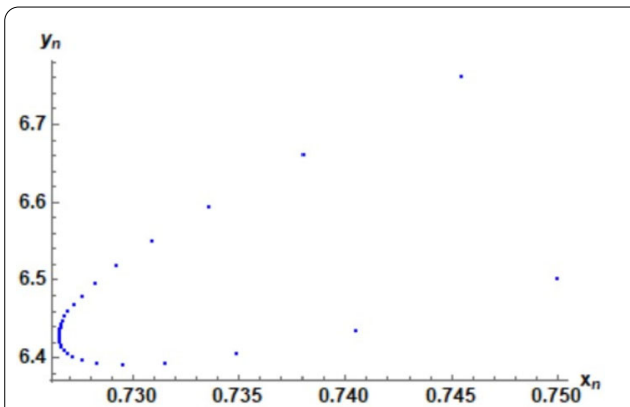

(a) $h=0.36$

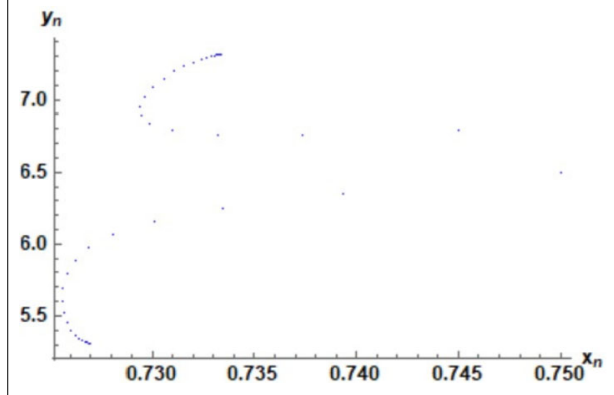

(c) $h=0.40$

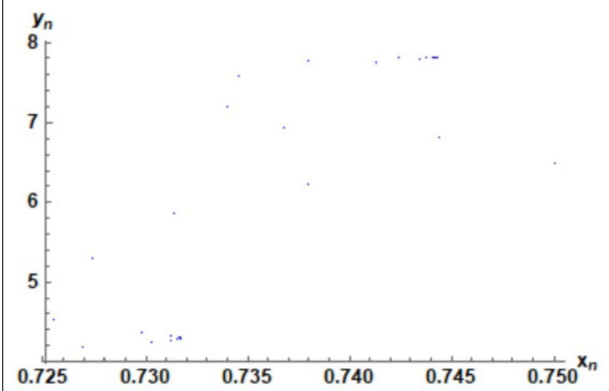

(e) $h=0.45$

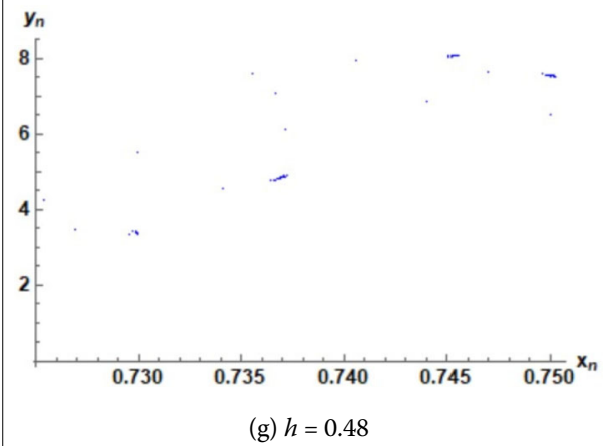

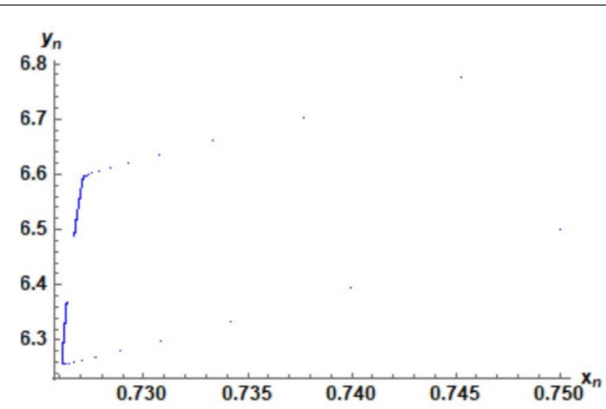

(b) $h=0.3805$

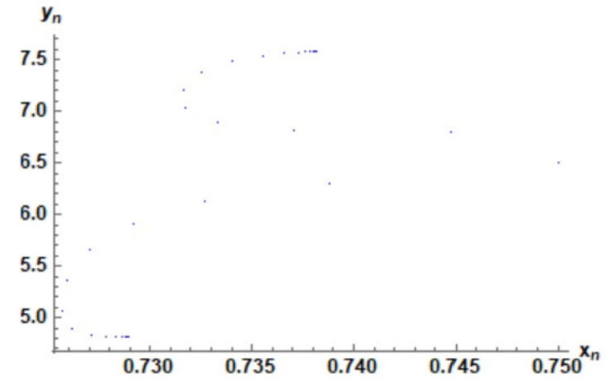

(d) $h=0.42$

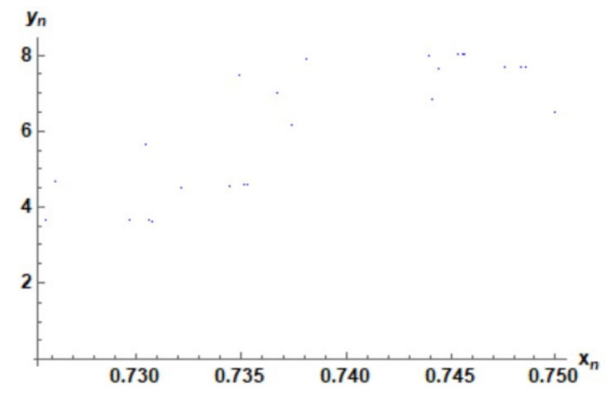

(f) $h=0.47$

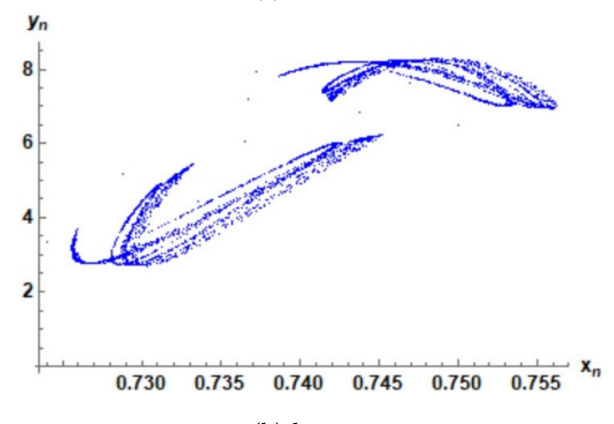

(h) $h=0.50$

Figure 4 Phase portraits for model (2) at different values of $h$ with $(a, b, c, d, \alpha, \beta)=(0.4,2,5.4,0.113,0.012,6)$ with initial conditions $\left(x_{0}, y_{0}\right)=(0.75,6.5)$

Then linearized form of (28) approximated at a positive equilibrium point $\left(x^{*}, y^{*}\right)=$ $(0.384976,1.33584)$ can be written as

$$
\left[\begin{array}{c}
x_{n+1}-0.384976 \\
y_{n+1}-1.33584
\end{array}\right] \approx A\left[\begin{array}{c}
x_{n}-0.384976 \\
y_{n}-1.33584
\end{array}\right]+B[h-0.575],
$$




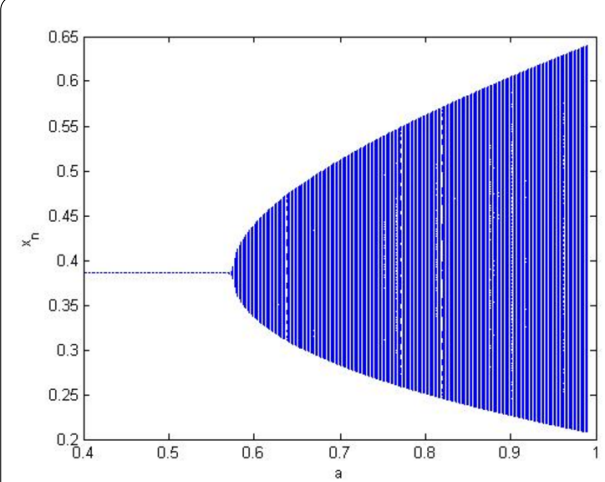

(a) Bifurcation diagram of $x_{n}$

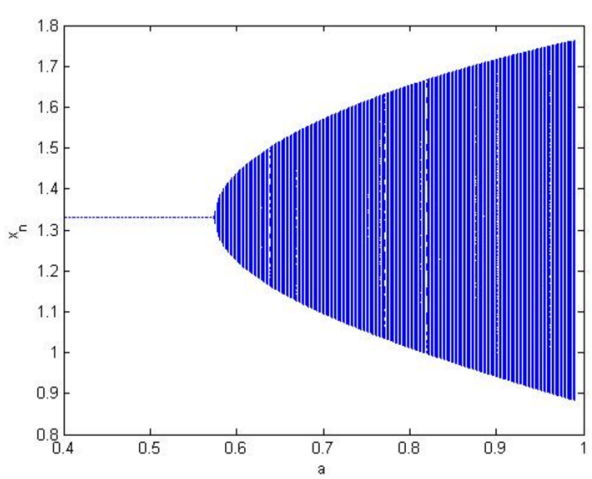

(b) Bifurcation diagram of $y_{n}$

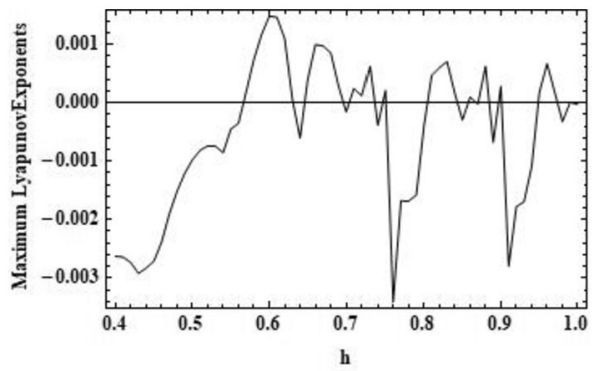

(c) Maximum Lyapunov exponents

Figure $5 \mathrm{MLE}$ and bifurcation diagrams for model (2) in the interval $0.4 \leq h \leq 0.99$ with parameters $(a, b, c, d, \alpha, \beta, h)=(0.001,0.75,0.224,0.29,0.19,0.5731)$ and initial conditions $\left(x_{0}, y_{0}\right)=(0.38564,1.33674)$

where

$$
A=\left[\begin{array}{cc}
1.096121758 & -0.1022448706 \\
0.4441379310 & 0.871200
\end{array}\right], \quad B=\left[\begin{array}{c}
0.0003773848246 \\
0
\end{array}\right] .
$$

Then it follows that $C=[B: A B]=\left[\begin{array}{cc}0.0003773848246 & 0.000413659717383074 \\ 0 & 0.000167610915188642\end{array}\right]$ is of rank 2 .

Taking $h=\left(h_{o}-k_{1}\left(x_{n}-x^{*}\right)-k_{2}\left(y_{n}-y^{*}\right)\right)$, where $\mathrm{K}=\left(k_{1}, k_{2}\right)$ is the gain matrix, the respective controlled system is given as

$$
\begin{aligned}
x_{n+1}= & x_{n}+\left(h_{o}-k_{1}\left(x_{n}-x^{*}\right)-k_{2}\left(y_{n}-y^{*}\right)\right)\left[x_{n}\left(1-x_{n}\right)-\frac{x_{n} y_{n}}{\frac{x_{n}^{2}}{0.19}+x_{n}+1}\right. \\
& \left.-\frac{0.001 x_{n}}{x_{n}+0.75}\right], \\
y_{n+1}= & y_{n}+\left(h_{o}-k_{1}\left(x_{n}-x^{*}\right)-k_{2}\left(y_{n}-y^{*}\right)\right)\left[0.224 y_{n}\left(1-0.29 \frac{y_{n}}{x_{n}}\right)\right] .
\end{aligned}
$$

By simple calculations, we get $\alpha_{1}=-1.967321758, \quad \alpha_{2}=1.000352101$, $\beta_{1}=0.0003773848246 k_{1}-1.967321758$, and $\beta_{2}=1.000352101-0.00032877765 k_{1}+$ $0.00016761091 k_{2}$. Next it is easy to see that $M=\left(\begin{array}{cc}-1.967321758 & 1 \\ 1 & 0\end{array}\right)$ and $T=$ $\left(\begin{array}{c}-0.000328777650 .000377384824 \\ 0.000167610915\end{array}\right)$. For the purpose of simplification, we fix $k_{2}=k$ and $k_{1}=0.1$ as an arbitrary parameter in controlled system (29), then the characteristic equation of 


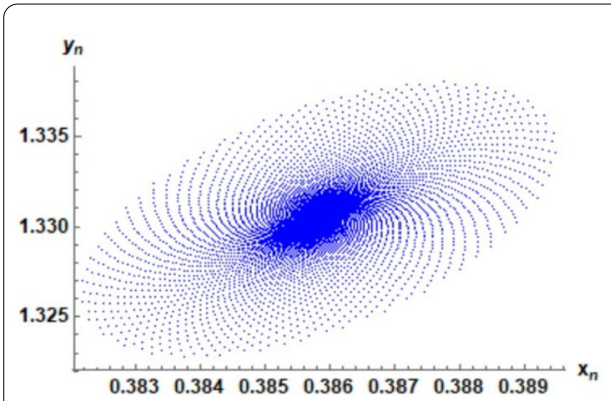

(a) $h=0.56$

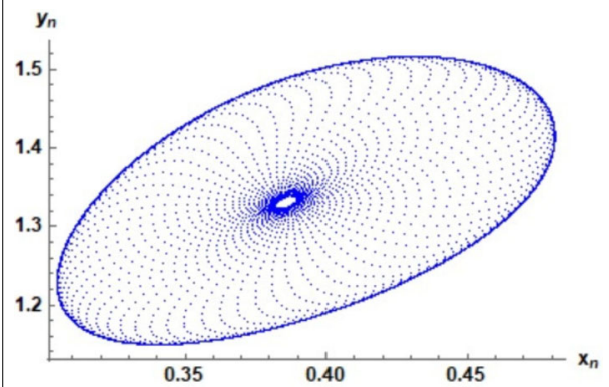

(c) $h=0.65$

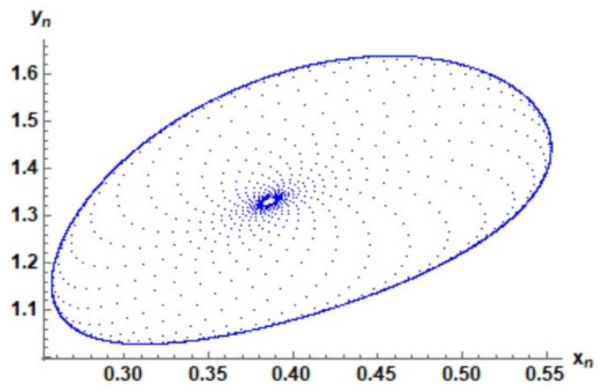

(e) $h=0.78$

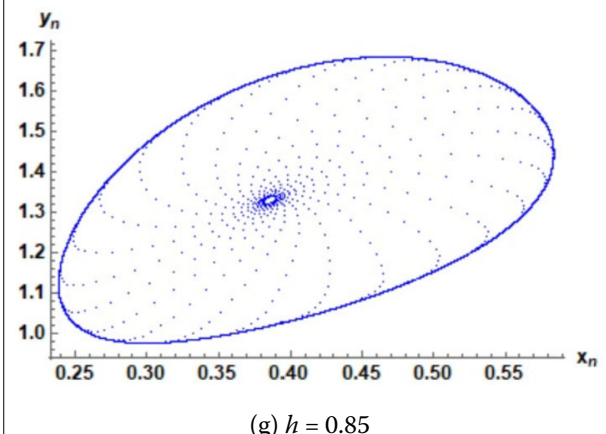

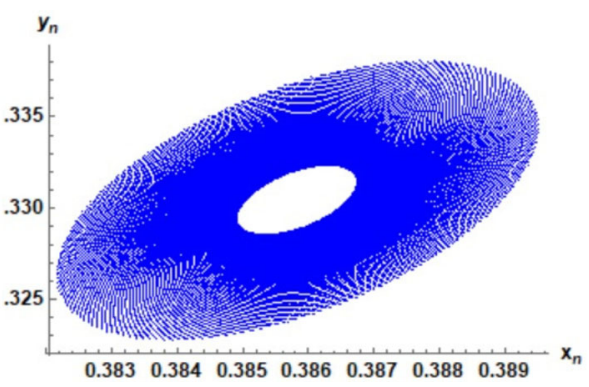

(b) $h=0.5731$

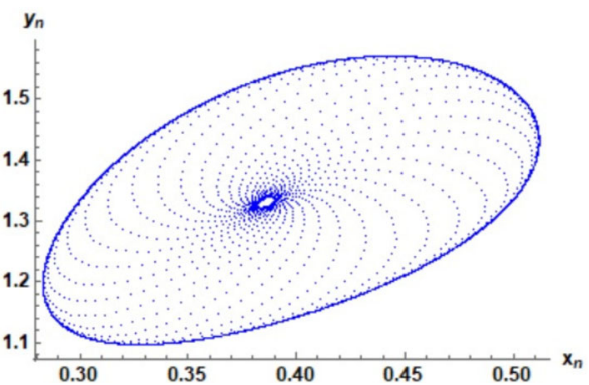

(d) $h=0.70$

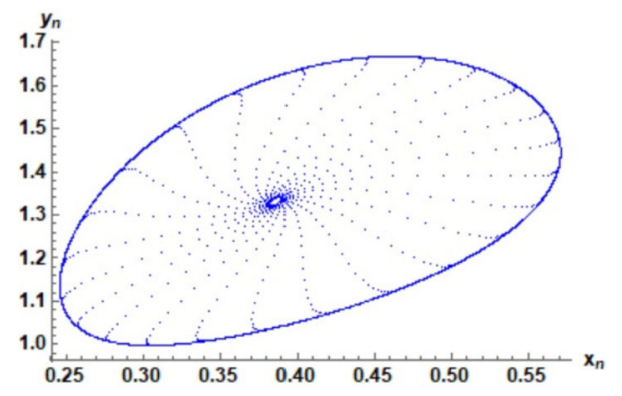

(f) $h=0.75$

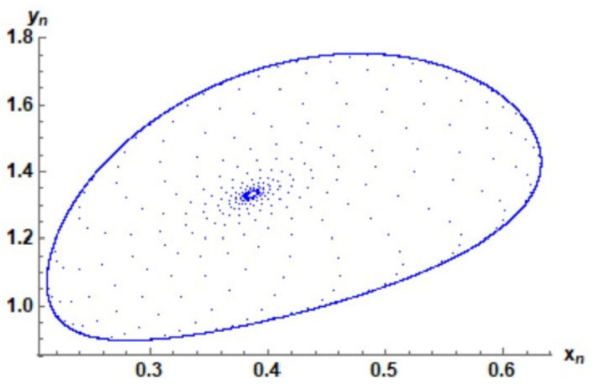

(h) $h=0.97$

Figure 6 Phase portraits for model (2) at different values of $h$ with

$(a, b, c, d, \alpha, \beta)=(0.001,0.75,0.224,0.29,0.19,1)$ and initial conditions $\left(x_{0}, y_{0}\right)=(0.38564,1.33674)$

Jacobian matrix (29) is given by

$$
\begin{aligned}
& \lambda^{2}+0.000473780 \lambda k-1.967284020 \lambda+0.000651671 k \\
& \quad+0.0000093731 k^{2}+1.00031922=0 .
\end{aligned}
$$




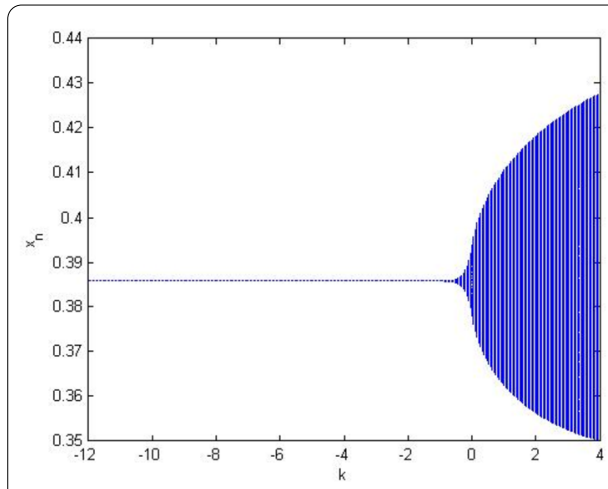

(a) Bifurcation diagram of $x_{n}$

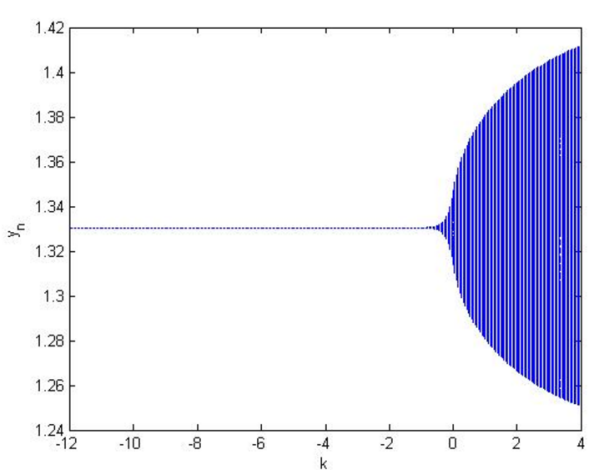

(b) Bifurcation diagram of $y_{n}$

Figure 7 Bifurcation diagrams for controlled system (29)

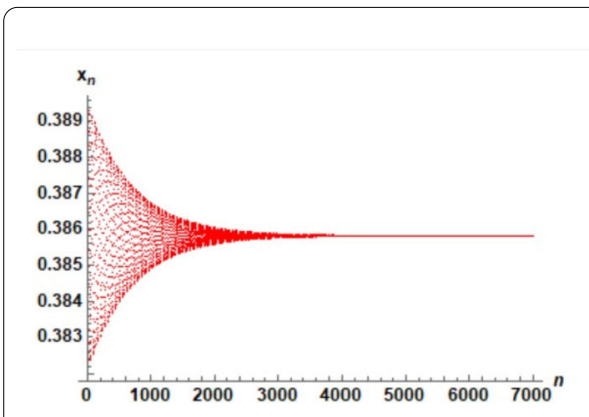

(a) Plot of $x_{n}$ for controlled system (29)

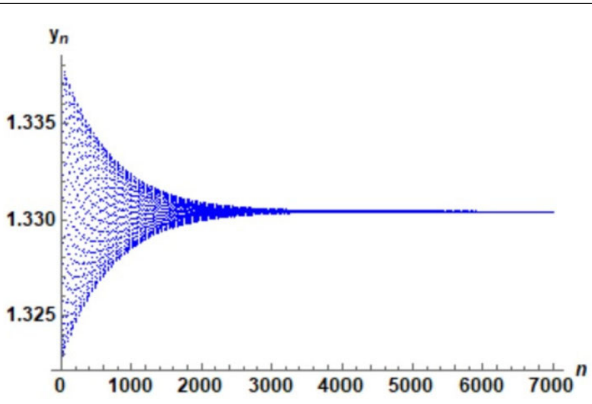

(b) Plot of $y_{n}$ for controlled system (29)

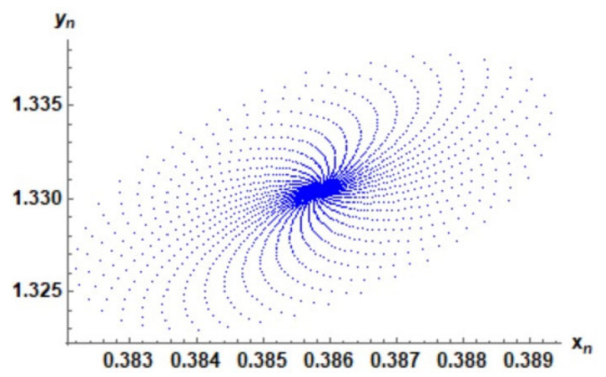

(c) Phase portrait for controlled system (29)

Figure 8 Plots for controlled system (29)

In this case the positive equilibrium of control system (29) is locally asymptotically stable for -69.03215478$)<k<-0.4933532528$. The bifurcation diagrams for controlled system (29) are shown in Fig. 7 by taking $k_{1}=0.1$ and $-12<k_{2}<4$. Next we take $k_{1}=0.1$ and $k_{2}=-10$ for controlled system (29), then the plots $x_{n}$ and $y_{n}$ are shown in Figs. 8(a) and (b) and the phase portrait is shown in Fig. 8(c).

Example 6.4 We apply the pole-placement control strategy for period-doubling bifurcation to system (2) and select the nominal value $h_{0}=0.47$ that belongs to the chaotic region $[0.3805,0.5]$. At $(a, b, c, d, \alpha, \beta)=(0.4,2,5.4,0.113,0.012,6)$ the unique positive equilibrium point $\left(x^{*}, y^{*}\right)=(0.730715,3.62792)$ of system (2) is unstable. In this case system (22) can 
be written as

$$
\begin{aligned}
& x_{n+1}=x_{n}+h\left[x_{n}\left(1-x_{n}\right)-\frac{x_{n} y_{n}}{\frac{x_{n}^{2}}{0.012}+x_{n}+6}-\frac{0.4 x_{n}}{x_{n}+2}\right]=f\left(x_{n}, y_{n}, h\right), \\
& y_{n+1}=y_{n}+h\left[5.4 y_{n}\left(1-0.113 \frac{y_{n}}{x_{n}}\right)\right]=g\left(x_{n}, y_{n}, h\right) .
\end{aligned}
$$

Then linearized form (31) approximated at a positive equilibrium point $\left(x^{*}, y^{*}\right)=$ $(0.730715,3.62792)$ can be written as

$$
\left[\begin{array}{c}
x_{n+1}-0.730715 \\
y_{n+1}-3.62792
\end{array}\right] \approx A\left[\begin{array}{c}
x_{n}-0.730715 \\
y_{n}-3.62792
\end{array}\right]+B[h-0.47],
$$

where

$$
A=\left[\begin{array}{cc}
0.7772896532 & -0.006704319996 \\
22.46017699 & -1.538
\end{array}\right], \quad B=\left[\begin{array}{c}
-0.0025073679 \\
0
\end{array}\right] .
$$

Then it follows that $C=[B: A B]=\left[\begin{array}{cc}-0.0025073679 & -0.00194895112543581 \\ 0 & -0.0563159268130446\end{array}\right]$ is of rank 2 .

Taking $h=\left(h_{o}-k_{1}\left(x_{n}-x^{*}\right)-k_{2}\left(y_{n}-y^{*}\right)\right)$, where $K=\left(k_{1}, k_{2}\right)$ is the gain matrix, the respective controlled system is given as

$$
\begin{aligned}
x_{n+1}= & x_{n}+\left(h_{o}-k_{1}\left(x_{n}-x^{*}\right)-k_{2}\left(y_{n}-y^{*}\right)\right)\left[x_{n}\left(1-x_{n}\right)-\frac{x_{n} y_{n}}{\frac{x_{n}^{2}}{0.012}+x_{n}+6}\right. \\
& \left.-\frac{0.4 x_{n}}{x_{n}+2}\right], \\
y_{n+1}= & y_{n}+\left(h_{o}-k_{1}\left(x_{n}-x^{*}\right)-k_{2}\left(y_{n}-y^{*}\right)\right)\left[5.4 y_{n}\left(1-0.113 \frac{y_{n}}{x_{n}}\right)\right] .
\end{aligned}
$$

By simple calculations, we get $\alpha_{1}=0.7607103468, \alpha_{2}=-1.044891273, \beta_{1}=$ $-0.0025073679 k_{1}+0.7607103468$, and $\beta_{2}=-1.04489127-0.00385633183 k_{1}-$ $0.0563159268 k_{2}$. Next it is easy to see that $M=\left(\begin{array}{c}0.76071034681 \\ 1\end{array}\right)$ and $T=$ $\left(\begin{array}{cc}-0.00385633183 & -0.00250736790 \\ -0.0563159268 & 0\end{array}\right)$. For the purpose of simplification, we fix $k_{2}=k$ and $k_{1}=0.1$ as an arbitrary parameter in controlled system (32), then the characteristic equation of the Jacobian matrix of (32) is given by

$$
\begin{aligned}
\lambda^{2}-16.6734242 \lambda k & +0.760459609 \lambda+7.974513942 k+26.45038149 k^{2} \\
& -1.045276906=0 .
\end{aligned}
$$

The positive equilibrium of control system (32) is locally asymptotically stable for $0.030673<k<0.165560$. The bifurcation diagrams for controlled system (32) are shown in Fig. 9 by taking $k_{1}=0.1$ and $-0.02<k_{2}<0.14$. Next we take $k_{2}=0.06$ and $k_{1}=0.1$ for controlled system (32), then the plots $x_{n}$ and $y_{n}$ are shown in Figs. 10(a) and (b) and the phase portrait is shown in Fig. 10(c).

Example 6.5 Now, choose the parameters $(a, b, c, d, \alpha, \beta)=(0.4,2,5.4,0.113,0.012,6)$ and $h=0.3805$ and the initial condition and $\left(x_{0}, y_{0}\right)=(0.75,6.5)$. Then the controlled system 


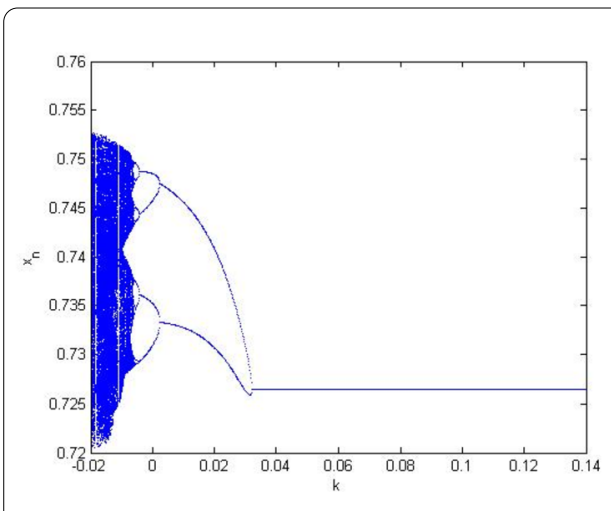

(a) Bifurcation diagram of $x_{n}$

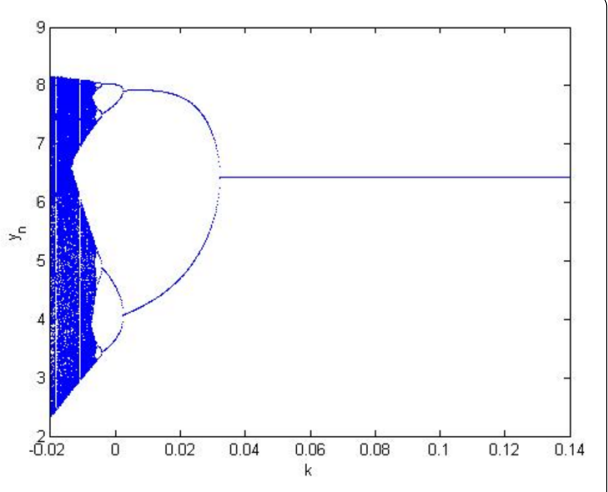

(b) Bifurcation diagram of $y_{n}$

Figure 9 Bifurcation diagrams for controlled system (32)

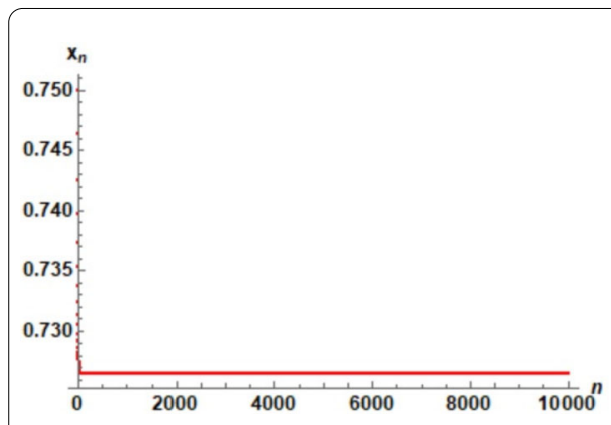

(a) Plot of $x_{n}$ for controlled system (32)

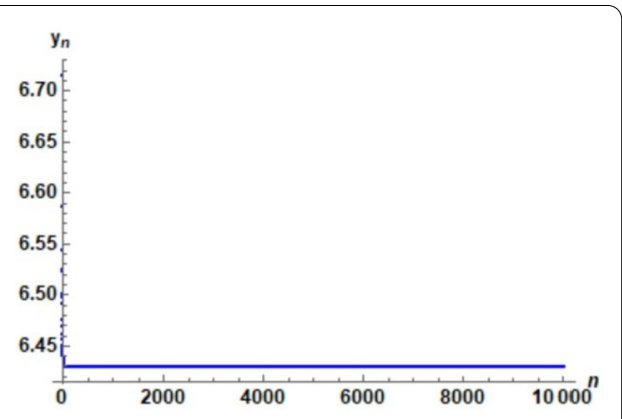

(b) Plot of $y_{n}$ for controlled system (32)

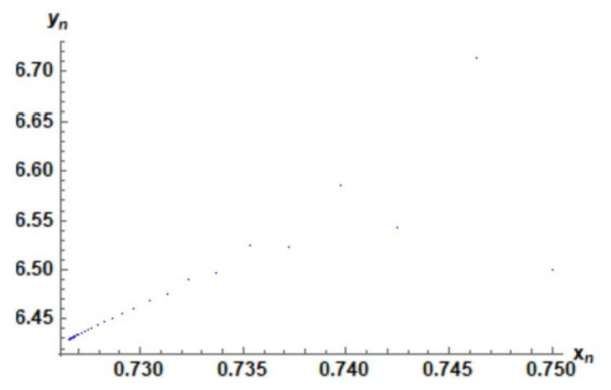

(c) Phase portrait for controlled system (32)

Figure 10 Plots and phase portrait for controlled system (32)

using hybrid control strategy is given by

$$
\begin{aligned}
& x_{n+1}=\rho\left[x_{n}+0.3805\left(x_{n}\left(1-x_{n}\right)-\frac{x_{n} y_{n}}{\frac{x_{n}^{2}}{0.012}+x_{n}+1}-\frac{0.4 x_{n}}{x_{n}+2}\right)\right]+(1-\rho) x_{n}, \\
& y_{n+1}=\rho\left[y_{n}+0.3805\left(5.4 y_{n}\left(1-\frac{0.113 y_{n}}{x_{n}}\right)\right)\right]+(1-\rho) y_{n} .
\end{aligned}
$$

The Jacobian matrix of (34) is

$$
J=\left[\begin{array}{cc}
-0.1946040346 \rho+1 & -0.005321678323 \rho \\
18.18318584 \rho & -2.05470 \rho+1
\end{array}\right] .
$$




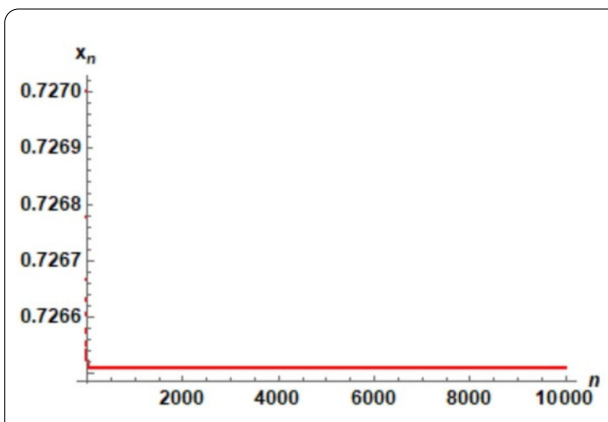

(a) Plot of $x_{n}$ for controlled system (34)

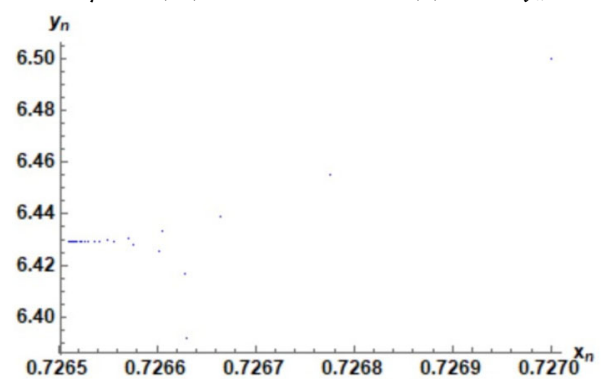

(c) Phase portrait for controlled system (34)

Figure 11 Plots and phase portrait for controlled system (34) with $(a, b, c, d, \alpha, \beta)=(0.4,2,5.4,0.113,0.012,6)$ and $h=0.3803$ and initial conditions $\left(x_{0}, y_{0}\right)=(0.75,6.5)$

The characteristic polynomial equation of (35) is

$$
P(\lambda)=\lambda^{2}+(2.249304035 \rho-2) \lambda+1-2.249304035 \rho+0.4966179758 \rho^{2}=0 .
$$

Under the Jury condition, the roots of (36) lie in a unit open disk if $0<\rho<1$. Thus, the period-doubling bifurcation is completely controlled in the largest allowable interval $\rho \in 0.9994323$. For $\rho=0.90$, the plots of $x_{n}, y_{n}$ and the phase portrait of controlled system (34) are shown in Figs. 11(a), (b), and (c), respectively.

Example 6.6 We again select the parameters $(a, b, c, d, \alpha, \beta)=(0.001,0.75,0.224,0.29$, $0.19,1)$ and $h=0.5731$ and the initial condition $\left(x_{0}, y_{0}\right)=(0.38564,1.33674)$, then system (2) undergoes NSB corresponding to the parametric values, we have the following controlled system:

$$
\begin{aligned}
& x_{n+1}=\rho\left[x_{n}+0.5731\left(x_{n}\left(1-x_{n}\right)-\frac{x_{n} y_{n}}{\frac{x_{n}^{2}}{0.19}+x_{n}+1}-\frac{0.001 x_{n}}{x_{n}+0.75}\right)\right]+(1-\rho), \\
& y_{n+1}=\rho\left[y_{n}+0.5731\left(0.224 y_{n}\left(1-\frac{0.29 y_{n}}{x_{n}}\right)\right)\right]+(1-\rho) y_{n} .
\end{aligned}
$$

The Jacobian matrix of (37) is given by

$$
J=\left[\begin{array}{cc}
0.095529046 \rho+1 & -0.1019247393 \rho \\
0.4426703448 \rho & -0.1283744 \rho+1
\end{array}\right] .
$$




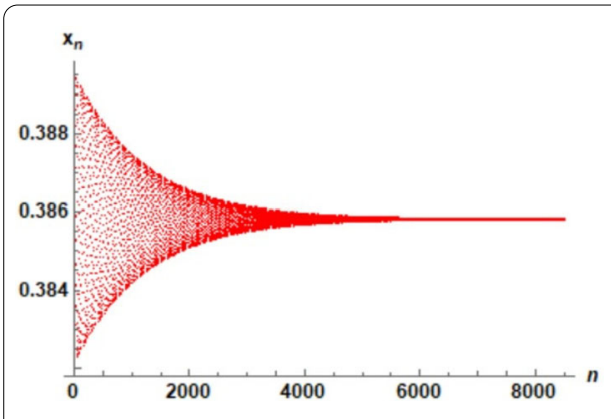

(a) Plot of $x_{n}$ for controlled system (37)

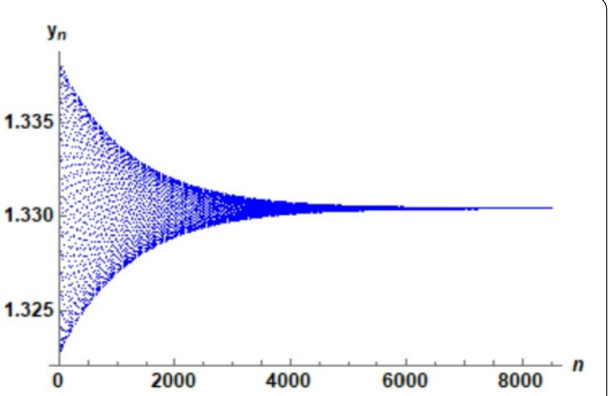

(b) Plot of $y_{n}$ for controlled system (37)

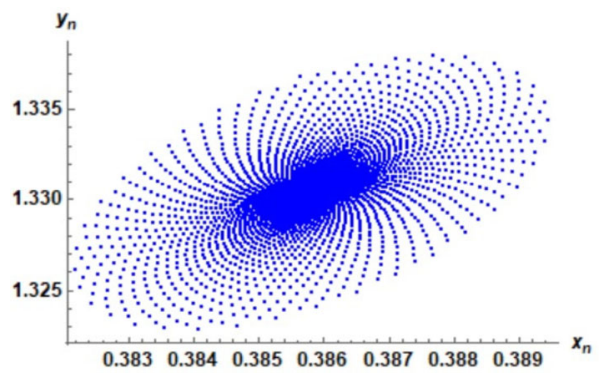

(c) Phase portrait for controlled system (37)

Figure 12 Plots and phase portrait for controlled system (37) with $(a, b, c, d, \alpha, \beta)=(0.001,0.75,0.224,0.29,0.19,1)$ and $h=0.5731$ and initial conditions $\left(x_{0}, y_{0}\right)=(0.38564,1.33674)$

The characteristic polynomial equation of (38) is

$$
P(\lambda)=\lambda^{2}+(0.032845354 \rho-2) \lambda+1-0.032845354 \rho+0.03285557553 \rho^{2}=0 .
$$

Under the Jury condition, the roots of (39) lie in a unit open disk if and only if $0<\rho<1$. Thus NSB is entirely controlled in the largest admissible interval for $\rho \in 0.9996888951$. For $\rho=0.95$, the plots and phase portrait of controlled system (37) are shown in Fig. 12.

\section{Concluding remarks}

We studied the qualitative behavior of a modified Leslie-Gower predator-prey model and achieved the results for stability of equilibrium points. In order to support the complexity in system (2), the presence of period-doubling and Neimark-Sacker bifurcation for the fixed point is verified mathematically, further numerically simulations are performed. Using these simulations, we showed that system (2) goes through PDB and NSB for the vast range of bifurcation parameters $h$. Chaos control is discussed through the implementation of pole-placement and hybrid feedback control methods. It is clear from our numerical observations that, for an extensive range of parameters, stability can be rebuilt through the OGY method and the hybrid control method. The OGY method is based on feedback control methodology, whereas hybrid control depends on feedback control and parameter perturbation. The computation of maximum Lyapunov exponents proved the presence of chaotic behavior of the system. 


\section{Acknowledgements}

The authors are grateful to referees for their careful reading, suggestions, and valuable comments which have improved the paper substantially.

Funding

No funding is available.

Availability of data and materials

Not applicable in our research.

\section{Competing interests}

The authors declare that they have no competing interests.

Authors' contributions

All authors contributed equally to the writing of this paper. All authors read and approved the manuscript.

\section{Author details}

${ }^{1}$ School of Natural Sciences, National University of Sciences and Technology, Islamabad, Pakistan. ${ }^{2}$ NUST Institute of Civil Engineering, School of Civil and Environmental Engineering, National University of Sciences and Technology, Islamabad, Pakistan. ${ }^{3}$ Department of Mathematics, University of Poonch Rawalakot, Rawalakot, Pakistan.

\section{Publisher's Note}

Springer Nature remains neutral with regard to jurisdictional claims in published maps and institutional affiliations.

Received: 21 September 2019 Accepted: 8 January 2020 Published online: 21 January 2020

\section{References}

1. Ahmad, S.: On the non-autonomous Volterra-Lotka competition equations. Proc. Am. Math. Soc. 117(1), 199-204 (1993)

2. Zhou, Z., Zou, X.: Stable periodic solutions in a discrete periodic logistic equation. Appl. Math. Lett. 16(2), 165-171 (2003)

3. Tang, X., Zou, X.: On positive periodic solutions of Lotka-Volterra competition systems with deviating arguments. Proc. Am. Math. Soc. 134(10), 2967-2974 (2006)

4. Liu, X.: A note on the existence of periodic solutions in discrete predator-prey models. Appl. Math. Model. 34(9), $2477-2483(2010)$

5. Liu, X., Xiao, D.: Complex dynamic behaviors of a discrete-time predator-prey system. Chaos Solitons Fractals 32(1), 80-94 (2007)

6. Agiza, H.N., Elabbasy, E.M., El-Metwally, H., Elsadany, A.A.: Chaotic dynamics of a discrete prey-predator model with Holling type II. Nonlinear Anal., Real World Appl. 10(1), 116-129 (2009)

7. Nusse, H.E., Yorke, J.A.: Border-collision bifurcations for piece wise smooth one-dimensional maps. Int. J. Bifurc. Chaos Appl. Sci. Eng. 5(01), 189-207 (1995)

8. Gao, S., Chen, L.: The effect of seasonal harvesting on a single-species discrete population model with stage structure and birth pulses. Chaos Solitons Fractals 24(4), 1013-1023 (2005)

9. Ali, I., Saeed, U., Din, Q.: Bifurcation analysis and chaos control in discrete-time system of three competing species. Arab. J. Math. 8(1), 1-4 (2019)

10. Saeed, U., Ali, I., Din, Q.: Neimark-Sacker bifurcation and chaos control in discrete-time predator-prey model with parasites. Nonlinear Dyn. 94(4), 2527-2536 (2018)

11. Din, Q., Saeed, U.: Bifurcation analysis and chaos control in a host-parasitoid model. Math. Methods Appl. Sci. 40(14) 5391-5406 (2017)

12. May, R.M., Oster, G.F.: Bifurcations and dynamic complexity in simple ecological models. Am. Nat. 110(974), 573-599 (1976)

13. Lotka, A.J.: Elements of physical biology. Sci. Prog. 20th Century 21(82), 341-343 (1926)

14. Lotka, A.J.: Fluctuations in the abundance of a species considered mathematically. Nature 119(2983), 12 (1927)

15. Leslie, P.H., Gower, J.C.: The properties of a stochastic model for the predator-prey type of interaction between two species. Biometrika 47(3/4), 219-234 (1960)

16. Wollkind, D.J., Logan, J.A.: Temperature-dependent predator-prey mite ecosystem on apple tree foliage. J. Math. Biol. 6(3), 265-283 (1978)

17. Wollkind, D.J., Collings, J.B., Logan, J.A.: Metastability in a temperature-dependent model system for predator-prey mite outbreak interactions on fruit trees. Bull. Math. Biol. 50(4), 379-409 (1988)

18. Aziz-Alaoui, M.A., Okiye, M.D.: Boundedness and global stability for a predator-prey model with modified Leslie-Gower and Holling-type II schemes. Appl. Math. Lett. 16(7), 1069-1075 (2003)

19. Huang, J.C., Xiao, D.M.: Analyses of bifurcations and stability in a predator-prey system with Holling type-IV functional response. Acta Math. Appl. Sin. 20(1), 167-178 (2004)

20. Cai, Y., Gui, Z., Zhang, X., Shi, H., Wang, W.: Bifurcations and pattern formation in a predator-prey model. Int. J. Bifurc. Chaos 28(11), $1850140(2018)$

21. Zhang, H., Cai, Y., Fu, S., Wang, W.: Impact of the fear effect in a prey-predator model incorporating a prey refuge. Appl. Math. Comput. 356, 328-337 (2019)

22. Wang, J., Cai, Y., Fu, S., Wang, W.: The effect of the fear factor on the dynamics of a predator-prey model incorporating the prey refuge. Chaos 29, 083109 (2019)

23. Turchin, P.: Complex Population Dynamics, p. 35. Princeton University Press, Princeton (2003) 
24. Brauer, F., Castillo-Chavez, C., Castillo-Chavez, C.: Mathematical Models in Population Biology and Epidemiology, p. 40. Springer, New York (2001)

25. Zhang, Z., Upadhyay, R.K., Datta, J.: Bifurcation analysis of a modified Leslie-Gower model with Holling type-IV functional response and nonlinear prey harvesting. Adv. Differ. Equ. 2018(1), 127 (2018)

26. He, Z., Lai, X.: Bifurcation and chaotic behavior of a discrete-time predator-prey system. Nonlinear Anal., Real World Appl. 12(1), 403-417 (2011)

27. Jing, Z., Yang, J.: Bifurcation and chaos in discrete-time predator-prey system. Chaos Solitons Fractals 27(1), 259-277 (2006)

Submit your manuscript to a SpringerOpen ${ }^{\circ}$ journal and benefit from:

- Convenient online submission

- Rigorous peer review

- Open access: articles freely available online

- High visibility within the field

- Retaining the copyright to your article

Submit your next manuscript at $\gg$ springeropen.com 